\title{
Simplicial Relative Cohomology Rings of Digital Images
}

\author{
Ismet Karaca ${ }^{1, *}$ and Gulseli Burak ${ }^{2}$ \\ ${ }^{1}$ Department of Mathematics, Ege University, 35100 Izmir, Turkey \\ ${ }^{2}$ Department of Mathematics, Pamukkale University, 20070 Denizli, Turkey
}

Received: 5 Sep. 2013, Revised: 3 Dec. 2013, Accepted: 4 Dec. 2013

Published online: 1 Sep. 2014

\begin{abstract}
The first goal of this paper is to show that the relative cohomology groups of digital images are determined algebraically by the relative homology groups of digital images. Then we state simplicial cup product for digital images and use it to establish ring structure of digital cohomology. Furthermore we give a method for computing the cohomology ring of digital images and give some examples concerning cohomology ring.
\end{abstract}

Keywords: Digital simplicial relative cohomology group, cup product, cohomology ring.

\section{Introduction}

In general calculating homology is not enought for determining differences between topological spaces. The cup product on cohomology is finer invariant. The cup product makes the cohomology group of a space into a ring. The ring structure from the cup product is an important advantage of cohomology theory over homology. While the homology groups of a space are equal to the cohomology groups, the ring structure on the cohomologies of the space is different. Then cup product can be used to distinguish the spaces.

Cohomology groups are determined algebraically by the homology groups. We will define the relative cohomology groups of digital images and show that these satisfy basic properties very much like those for the relative homology of digital images.

Althought basic properties of cohomology theory are similar to homology theory, there are some differences between them. One of the differences is that cohomology group is contravariant functor while homology group is covariant. Contravariance leads to additional structures in cohomology. These new structures are finer invariants of homotopy type and enable us to distinguish between topological spaces what are called cup products and cohomology operations.
Many researchers(Rosenfeld [24], Kopperman [20], Kong [19], Malgouyres [21], Boxer [4, 5, 6, 7,9], Han [11, 12], Karaca [1,10]) have contributed to digital topology with their studies. They wish to characterize the properties of digital images with tools from Algebraic Topology. Their results play an important role in our study.

Arslan, Karaca and Oztel [1], define simplicial homology group of a digital image and give examples of simplicial homology groups of certain digital images. They also compute simplicial homology groups of $M S S_{18}$.

Gonzalez-Diaz and Real [15] have their 14-adjacency algorithm to compute cup products on the simplicial complex. The advantage of this method is tried via a small program visualizing the several steps. Gonzalez-Diaz, Jimenez and Medrano [16] introduce a method for computing cup products on cubical approximations. Their cup products are computed directly from the cubical complex. Gonzalez-Diaz, Lamar and Umble [17] present how to simplify the combinatorial structure of cubical complex and obtain a homeomorphic cellular complex with fewer cells. They introduce formulas for a diagonal approximation on a general polygon and use it to compute cup products on the cohomology. The algorithm offered their work can be applied to compute cup products on any polyhedral

\footnotetext{
*Corresponding author e-mail: ismet.karaca@ege.edu.tr
} 
approximation of an object embedded in 3-space.

Kaczynski and Mrozek [18] improve a process to compute cup product on cubical complexes for generating a cohomology ring algorithm. This method is useful to the topological analysis of high-dimensional data. Their theory is to construct a cohomology ring algorithm speeding up the algebraic calculations.

Ege and Karaca [10] propose a mathematical framework that can be used for defining cohomology of digital images. They state the Eilenberg-Steenrod axioms and the Universal Coefficient Theorem for this cohomology theory. They show that the Künneth formula for cohomology theory doesn't be hold in digital images. Moreover they state the cup product for digital images and prove its basic properties.

In Section 2, we review necessary backgrounds on digital topology. In next section we give definitions and theorems that are related to relative cohomology groups of digital images. In the last section we define the simplicial cup product and its general properties. Moreover we give examples about computing the cohomology ring of minimal simple surfaces $M S S_{18}^{\prime}$ and $M S S_{18}$.

\section{Preliminaries}

Let $\mathbb{Z}^{n}$ be the set of lattice points in the $n$-dimensional Euclidean space where $\mathbb{Z}$ is the set of integer. A (binary) digital image is a pair $(X, \kappa)$ where $X \subset \mathbb{Z}^{n}$ for some positive integer $n$ and $\kappa$ represents certain adjacency relation for the members of $X$. We use a variety of adjacency relations in the study of digital images.

Definition 21[5] Let $l, n$ be positive integers, $1 \leq l \leq n$ and distinct two points

$$
p=\left(p_{1}, p_{2}, \ldots, p_{n}\right), q=\left(q_{1}, q_{2}, \ldots, q_{n}\right) \in \mathbb{Z}^{n}
$$

$p$ and $q$ are $k_{l}$-adjacent if there are at most $l$ indices $i$ such that $\left|p_{i}-q_{i}\right|=1$ and for all other indices $j$ such that $\left|p_{i}-q_{i}\right| \neq 1, p_{j}=q_{j}$.

From Definition 2.1, we have the following;

- Two points $p$ and $q$ in $\mathbb{Z}$ are 2-adjacent if $|p-q|=1$.

- Two points $p$ and $q$ in $\mathbb{Z}^{2}$ are 8-adjacent if they are distinct and differ by at most 1 in each coordinate.

- Two points $p$ and $q$ in $\mathbb{Z}^{2}$ are 4-adjacent if they are 8-adjacent and differ in exactly one coordinate.

- Two points $p$ and $q$ in $\mathbb{Z}^{3}$ are 26-adjacent if they are distinct and differ by at most 1 in each coordinate.

- Two points $p$ and $q$ in $\mathbb{Z}^{3}$ are 18-adjacent if they are 26-adjacent and differ in at most two coordinate.

- Two points $p$ and $q$ in $\mathbb{Z}^{3}$ are 6-adjacent if they are

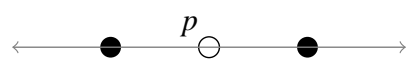

Fig. 1: 2-adjacent

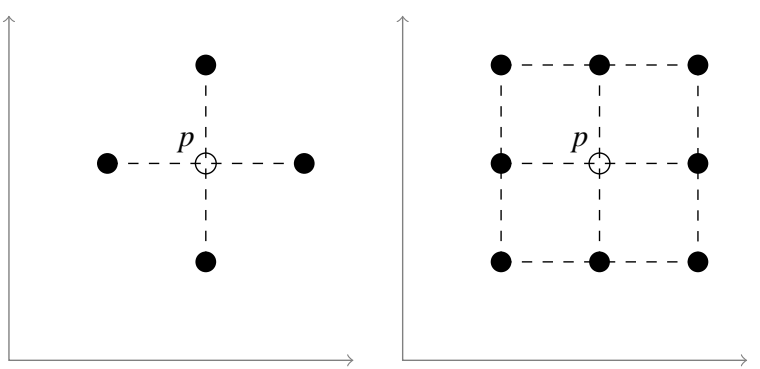

Fig. 2: 4-adjacent and 8-adjacent
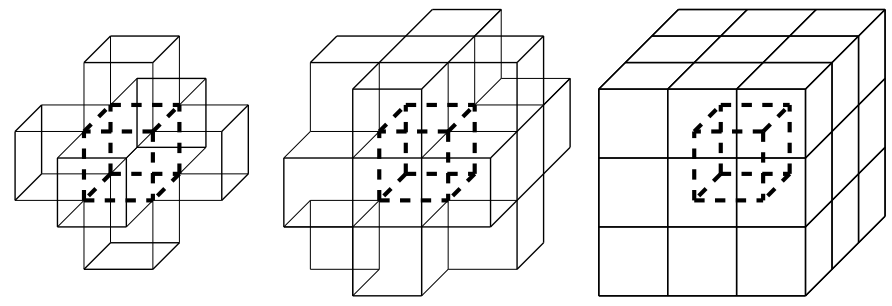

Fig. 3: 6-adjacent, 18-adjacent and 26-adjacent

18-adjacent and differ in exactly one coordinate.

Let $\kappa \in\{2,4,8,6,18,26\}$. A $\kappa$-neighbor of $p \in \mathbb{Z}^{n}$ is a point of $\mathbb{Z}^{n}$ that is $\kappa$-adjacent to $p$ [5]. The $\kappa$-neighborhood of $p$ is defined to be set

$$
N_{\kappa}(p)=\{q \mid q \text { is } \kappa \text {-adjacent to } p\} .
$$

Let $a, b \in \mathbb{Z}$ with $a<b$. A set of the form

$$
[a, b]_{\mathbb{Z}}=\{z \in \mathbb{Z} \mid a \leq z \leq b\}
$$

is called a digital interval [4].

Definition 22[14] Let $\kappa$ be an adjacency relation defined on $\mathbb{Z}^{n}$. A digital image $X \subset \mathbb{Z}^{n}$ is $\kappa$-connected if and only if for every pair of different points $x, y \in X$, there is a set $\left\{x_{0}, x_{1}, \ldots, x_{r}\right\}$ of points of a digital image $X$ such that $x=x_{0}, y=x_{r}$ and $x_{i}$ and $x_{i+1}$ are $\kappa$-neighbors where $i=0,1, \ldots, r-1$. A $\kappa$-component of a digital image $X$ is a maximal $\kappa$-connected subset of $X$.

Definition 23[5] Let $X \subset \mathbb{Z}^{n_{0}}$ and $Y \subset \mathbb{Z}^{n_{1}}$ be digital images with $\kappa_{0}$ adjacency and $\kappa_{1}$-adjacency respectively. Then the function $f: X \rightarrow Y$ is said to be $\left(\kappa_{0}, \kappa_{1}\right)$-continuous if for every $\kappa_{0}$-connected subset $U$ of $X, f(U)$ is a $\kappa_{1}$-connected subset of $Y$. We say that such a function is digitally continuous. 
Proposition 24[5] Let $X \subset \mathbb{Z}^{n_{0}}$ and $Y \subset \mathbb{Z}^{n_{1}}$ be digital images with $\kappa_{0}$-adjacency and $\kappa_{1}$-adjacency respectively. Then the function $f: X \rightarrow Y$ is said to be $\left(\kappa_{0}, \kappa_{1}\right)$-continuous if and only if for every pair of $\kappa_{0}$-adjacent points $\left\{x_{0}, x_{1}\right\}$ of $X$, either $f\left(x_{0}\right)=f\left(x_{1}\right)$ or $f\left(x_{0}\right)$ and $f\left(x_{1}\right)$ are $\kappa_{1}$-adjacent in $Y$.

A $(2, \kappa)$-continuous function $f:[0, m]_{\mathbb{Z}} \rightarrow X$ such that $f(0)=x$ and $f(m)=y$ is called $a$ digital $\kappa$-path from $x$ to $y$ in a digital image $X$ [5]. A digital image $X$ is digital $\kappa$-path connected if, for every $x, y \in X$, there exist a $\kappa$-path in $X$ from $x$ to $y$.

A simple closed $\kappa$-curve of $m \geq 4$ points in a digital image $X$ is a sequence

$$
\{f(0), f(1), \ldots, f(m-1)\}
$$

of images of the $\kappa$-path $f:[0, m-1]_{\mathbb{Z}} \rightarrow X$ such that $f(i)$ and $f(j)$ are $\kappa$-adjacent if and only if $j=i \pm 1 \bmod m$ [8].

Let $\left(X, \kappa_{0}\right) \subset \mathbb{Z}^{n_{0}}$ and $\left(Y, \kappa_{1}\right) \subset \mathbb{Z}^{n_{1}}$ be digital images. A function $f: X \rightarrow Y$ is $\left(\kappa_{0}, \kappa_{1}\right)$-isomorphism if $f$ is $\left(\kappa_{0}, \kappa_{1}\right)$-continuous and bijective and further $f^{-1}: Y \rightarrow X$ is $\left(\kappa_{1}, \kappa_{0}\right)$-continuous, in which case we denote $X \cong_{\left(\kappa_{0}, \kappa_{1}\right)} Y[7]$.

A point $x \in X$ is called a $\kappa$-corner if $x$ is $\kappa$-adjacent to two and only two points $y, z \in X$ such that $y$ and $z$ are $\kappa$ adjacent to each other [3]. The $\kappa$-corner $x$ is called simple if $y, z$ are not $\kappa$-corners and if $x$ is the only point $\kappa$-adjacent to both $y, z[2] . X$ is called a generalized simple closed $\kappa$ curve if what is obtained by removing all simple $\kappa$-corners of $X$ is a simple closed $\kappa$-curve [21]. For a $\kappa$-connected digital image $(X, \kappa)$ in $\mathbb{Z}^{n}$, we can state following

$$
|X|^{x}=N_{3^{n}-1}(x) \cap X .
$$

Definition 25[12] Let $(X, \kappa) \subset \mathbb{Z}^{n}$ be a digital image, $n \geq$ 3 , and $\bar{X}=\mathbb{Z}^{n}-X$. Then $X$ is called a closed $\kappa$-surface if it satisfies the following:

1.In that case $(\kappa, \bar{\kappa}) \in\left\{(\kappa, 2 n),\left(2 n, 3^{n}-1\right)\right\}$ and $\kappa \neq$ $3^{n}-2^{n}-1$, then;

-for each point $x \in X,|X|^{x}$ has exactly one $\kappa$-component $\kappa$-adjacent to $x$.

$-|\bar{X}|^{x}$ has exactly two $\bar{\kappa}$-components $\bar{\kappa}$-adjacent to $x$. (We denote by $C^{x x}$ and $D^{x x}$ these two components) -for any point $y \in N_{\kappa}(x) \cap X, N_{\bar{\kappa}}(y) \cap C^{x x} \neq \varnothing$ and $N_{\bar{\kappa}}(y) \cap D^{x x} \neq \varnothing$, where $N_{\kappa}$ means the $\kappa$-neighbors of $x$.

Furthermore, if a closed $\kappa$-surface $X$ does not have a simple $\kappa$-point, then $X$ is called simple.

2.In that case $(\kappa, \bar{\kappa})=\left(3^{n}-2^{n}-1,2 n\right)$, then

$-X$ is $\kappa$-connected,

-for each point $x \in X,|X|^{x}$ is a generalized simple closed $\kappa$-curve.

Furthermore, if the image $|X|^{x}$ is a simple closed $\kappa$ curve, then the closed $\kappa$-surface $X$ is called simple.

Example 26MSS $S_{18}$ and $M S S_{18}^{\prime}$ are minimal simple closed 18-surfaces.

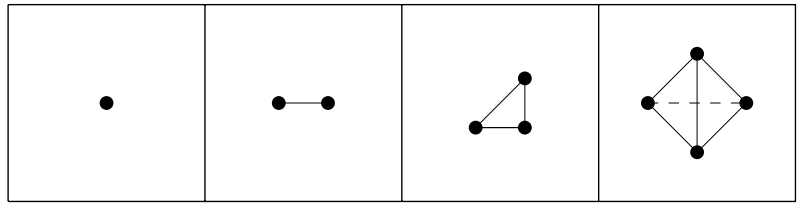

Fig. 4: (2,0), (2,1), (8,2) and (26,3)-simplexes

Definition 27[5] Let $\left(X, \kappa_{0}\right) \subset \mathbb{Z}^{n_{0}}$ and $\left(Y, \kappa_{1}\right) \subset \mathbb{Z}^{n_{1}}$ be digital images. Two $\left(\kappa_{0}, \kappa_{1}\right)$-continuous functions $f, g: X \rightarrow Y$ are said to be digitally $\left(\kappa_{0}, \kappa_{1}\right)$-homotopic in $Y$ if there is a positive integer $m$ and a function $H: X \times[0, m]_{\mathbb{Z}} \rightarrow Y$ such that for all $x \in X, H(x, 0)=f(x)$ and $H(x, m)=g(x)$; for all $x \in X$, the induced function $H_{x}:[0, m]_{\mathbb{Z}} \rightarrow Y$ defined by

$$
H_{x}(t)=H(x, t) \text { for all } t \in[0, m]_{\mathbb{Z}}
$$

is $\left(2, \kappa_{1}\right)$-continuous; and for all $t \in[0, m]_{\mathbb{Z}}$, the induced function $H_{t}: X \rightarrow Y$ defined by

$$
H_{t}(x)=H(x, t) \text { for all } x \in X,
$$

is $\left(\kappa_{0}, \kappa_{1}\right)$-continuous. The function $H$ is called a digital $\left(\kappa_{0}, \kappa_{1}\right)$-homotopy [2] between $f$ and $g$. A digital image $(X, \kappa)$ is said to be $\kappa$-contractible if its identity map is $(\kappa, \kappa)$-homotopic to a constant function $\bar{c}$ for some $c \in X$ where the constant function $\bar{c}: X \rightarrow X$ is defined by $\bar{c}(x)=$ $c$ for all $x \in X$.

For a digital image $(X, \kappa)$ and its subset $(A, \kappa)$, we call $(X, A)$ a digital image pair with $\kappa$-adjacency. Moreover, if $A$ is a singleton set $x_{0}$, then $\left(X, x_{0}\right)$ is called $a$ pointed digital image.

Definition 28[26] Let $S$ be a set of nonempty subset of a digital image $(X, \kappa)$. Then the members of $S$ are called simplexes of $(X, \kappa)$ if the following hold:

-If $p$ and $q$ are distinct points of $s \in S$, then $p$ and $q$ are $\kappa$-adjacent.

-If $s \in S$ and $\varnothing \neq t \subset s$, then $t \in S$.

A $m$-simplex is a simplex $S$ such that $|S|=m+1$. Let $P$ be a digital $m$-simplex. If $P^{\prime}$ is a nonempty proper subset of $P$, then $P^{\prime}$ is called a face of $P$. We write $\operatorname{Vert}(P)$ to denote the vertex set of $P$, namely, the set of all digital 0 -simplexes in $P$. A digital subcomplex $A$ of a digital simplicial complex $X$ with $\kappa$-adjacency is a digital simplicial complex contained in $X$ with $\operatorname{Vert}(A) \subset \operatorname{Vert}(X)$.

Let $(X, \kappa)$ be a finite collection of digital $m$-simplices, $0 \leq m \leq d$ for some non-negative integer $d$. $(X, \kappa)$ is called $\bar{a}$ finite digital simplicial complex [1] if the following statements hold:

-If $P$ belongs to $X$, then every face of $P$ also belongs to $X$. 
-If $P, Q \in X$, then $P \cap Q$ is either empty or a common face of $P$ and $Q$.

Definition 29[1] Let $(X, \kappa) \subset \mathbb{Z}^{n}$ be a digital simplicial complex. $(X, \kappa)$ called digital oriented simplicial complex if there is an ordering on the vertex set of $(X, \kappa)$.

Definition 210[1] Let $(X, \kappa) \subset \mathbb{Z}^{n}$ be a digital oriented simplicial complex with m-dimension. A homomorphism

$$
\partial_{q}: C_{q}^{\kappa}(X) \rightarrow C_{q-1}^{\kappa}(X)
$$

called the boundary operator. If $\sigma=\left[v_{0}, \ldots, v_{q}\right]$ is an oriented simplex with $0<q \leq m$, we define

$$
\partial_{q} \sigma=\partial_{q}\left[v_{0}, \ldots, v_{q}\right]=\sum_{i=0}^{q}(-1)^{i}\left[v_{0}, \ldots, \widehat{v}_{i}, \ldots, v_{q}\right]
$$

where the symbol $\widehat{v}_{i}$ means that the vertex $v_{i}$ is to be deleted from the array. Since $C_{q}^{\kappa}(X)$ is the trivial group for $q<0$, $m<q$ the operator $\partial_{q}$ is the trivial homomorphism for $q \leq$ $0, m<q$.

Proposition 211[1] For $m \geq q$, we have $\partial_{q-1} \circ \partial_{q}=0$.

Definition 212[9] Let $(X, \kappa) \subset \mathbb{Z}^{n}$ be a digital oriented simplicial complex with m-dimension. The kernel of $\partial_{q}: C_{q}^{\kappa}(X) \rightarrow C_{q-1}^{\kappa}(X)$ is called the group of $q$-cycles and denoted $Z_{q}^{\kappa}(X)$. The image of $\partial_{q+1}: C_{q+1}^{\kappa}(X) \rightarrow C_{q}^{\kappa}(X)$ is called the group of $q$-boundaries and is denoted $B_{q}^{\kappa}(X)$. We define the $q$ th simplicial homology group of $X$ by

$$
H_{q}^{\kappa}(X)=Z_{q}^{\kappa}(X) / B_{q}^{\kappa}(X) .
$$

Theorem 213[1] If $(X, \kappa) \subset \mathbb{Z}^{n}$ is a digital $\kappa$-path connected space then $H_{0}^{\kappa}(X) \cong \mathbb{Z}$.

Lemma 214 (The zig-zag lemma) [22] Suppose one is given simplicial complexes $\mathscr{C}=\left\{C_{p}, \partial_{C}\right\}, \mathscr{C}^{\prime}=\left\{C_{p}^{\prime}, \partial_{C^{\prime}}\right\}$ and $\mathscr{C}^{\prime \prime}=\left\{C_{p}^{\prime \prime}, \partial_{C^{\prime \prime}}\right\}$ and chain maps $\phi, \psi$ such that the sequence

$$
0 \longrightarrow \mathscr{C} \stackrel{\phi}{\longrightarrow} \mathscr{C}^{\prime} \stackrel{\psi}{\longrightarrow} \mathscr{C}^{\prime \prime} \longrightarrow 0
$$

is exact. Then there is a long exact homology sequence

$$
\begin{aligned}
& \cdots \longrightarrow H_{p}(\mathscr{C}) \stackrel{\phi_{*}}{\longrightarrow} H_{p}\left(\mathscr{C}^{\prime}\right) \stackrel{\psi_{*}}{\longrightarrow} H_{p}\left(\mathscr{C}^{\prime \prime}\right) \\
& \stackrel{\partial_{*}}{\longrightarrow} H_{p-1}(\mathscr{C}) \stackrel{\phi_{*}}{\longrightarrow} H_{p-1}\left(\mathscr{C}^{\prime}\right) \longrightarrow \cdots
\end{aligned}
$$

where $\partial_{*}$ is induced by the boundary operator in $\mathscr{C}^{\prime}$.

Definition 215[22] Let $(X, \kappa) \subset \mathbb{Z}^{n}$ be a digital simplicial complex; let $G$ be an abelian group. The digital simplicial cochain complex $\left(\mathscr{C}^{*}(X), \delta\right)$ is defined as follows. For any $q \in \mathbb{Z}$, the $q$-dimensional digital cochain group with coefficients in $G$, is the group

$$
C^{q, \kappa}(X ; G)=\operatorname{Hom}\left(C_{q}^{\kappa}(X), G\right) .
$$

The coboundary operator $\delta$ is defined to be the dual of the boundary operator $\partial: C_{q+1}^{\kappa}(X) \rightarrow C_{q}^{\kappa}(X)$. Thus

$$
C^{q+1, \kappa}(X ; G) \stackrel{\delta}{\longleftarrow} C^{q, \kappa}(X ; G)
$$

so that $\delta$ raises dimension by one. The abelian group $\mathrm{G}$ is omitted from the notation when it equals the group of integers. Elements of $C^{q, \kappa}(X)$ are called digital cochains and denoted either by $c^{q}$ or by $c^{*}$, if we don't need to specify their dimension $q$. The value of a digital cochain $c^{q}$ on a chain $d_{q}$ is denoted by $\left\langle c^{q}, d_{q}>\right.$. The $q$-th coboundary map $\delta^{q}: C^{q, \kappa}(X) \rightarrow C^{q+1, \kappa}(X)$ is the dual homomorphism of $\partial_{q+1}$ defined by

$$
<\delta^{q} c^{q}, d_{q+1}>=<c^{q}, \partial_{q+1} d_{q+1}>.
$$

Definition 216[22] The kernel of $\delta$ is called the group of cocycles and denoted by $Z^{q, \kappa}(X ; G)$, its image is called the group of coboundaries and denoted by $B^{q, \kappa}(X ; G)$.

Example 217 Let's compute the 0-cocycles of $M S C_{8}^{\prime}$.

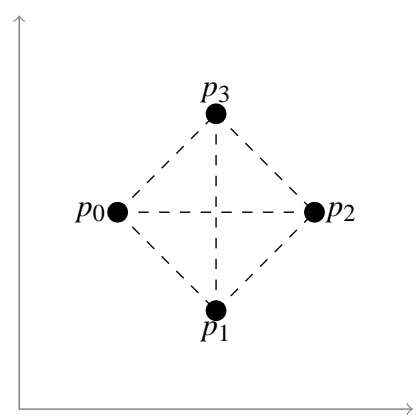

Fig. 5: $M S C_{8}^{\prime}$

Let $M S C_{8}^{\prime}=\left\{p_{0}=(1,2), p_{1}=(2,1), p_{2}=(3,2)\right.$, $\left.p_{3}=(2,3)\right\} \subset \mathbb{Z}^{2}$ and $p_{0}<p_{1}<p_{3}<p_{2}$.

0 -simplexes are $\left\langle p_{0}\right\rangle,\left\langle p_{1}\right\rangle,\left\langle p_{2}\right\rangle,\left\langle p_{3}\right\rangle$ and 1-simplexes are $e_{0}=\left\langle p_{0} p_{3}\right\rangle, e_{1}=\left\langle p_{3} p_{2}\right\rangle, e_{2}=\left\langle p_{1} p_{2}\right\rangle, e_{3}=\left\langle p_{0} p_{1}\right\rangle$.

We first find the 0-cochains. Since

$$
\begin{aligned}
& \partial_{1}\left(e_{0}\right)=p_{3}-p_{0} \\
& \partial_{1}\left(e_{1}\right)=p_{2}-p_{3} \\
& \partial_{1}\left(e_{2}\right)=p_{2}-p_{1} \\
& \partial_{1}\left(e_{3}\right)=p_{1}-p_{0}
\end{aligned}
$$

we get 0-cochains,

$$
\begin{aligned}
& \delta^{0} p_{0}^{*}=-e_{0}-e_{3} \\
& \delta^{0} p_{1}^{*}=-e_{2}+e_{3} \\
& \delta^{0} p_{2}^{*}=e_{1}+e_{2} \\
& \delta^{0} p_{3}^{*}=e_{0}-e_{1}
\end{aligned}
$$

Therefore we have that $p_{0}^{*}+p_{1}^{*}+p_{2}^{*}+p_{3}^{*}$ is a 0 -cocycle. 
Definition 218[22] The cohomology group of a digital image $(X, \kappa)$ with coefficients in $G$ is the group

$$
H^{q, \kappa}(X ; G)=Z^{q, \kappa}(X ; G) / B^{q, \kappa}(X ; G) .
$$

Theorem 219[10] If $(X, \kappa)$ is a single vertex, then

$$
H^{q, \kappa}(X)=\left\{\begin{array}{l}
\mathbb{Z}, q=0 \\
0, q \neq 0
\end{array}\right.
$$

Theorem 220 If $(X, \kappa) \subset \mathbb{Z}^{n}$ is a digital $\kappa$-path connected space then $H^{0, \kappa}(X) \cong \mathbb{Z}$.

Proof. Assume that 0 -simplexes of $X$ are $\left\langle p_{0}\right\rangle,\left\langle p_{1}\right\rangle, \ldots,\left\langle p_{n}\right\rangle$. We get the following sequence

$$
0 \stackrel{\delta^{-1}}{\longrightarrow} C^{0, \kappa}(X) \stackrel{\delta^{0}}{\longrightarrow} C^{1, \kappa}(X) .
$$

As the image of $\delta^{-1}$ is zero, $B^{0, \kappa}(X)=\{0\}$. Let us find $Z^{0, \kappa}(X)=\operatorname{Ker} \delta^{0}$. Let

$$
A=\left\{\sum_{i=0}^{n} k_{i} p_{i} \mid k_{i}=k, i=0,1, \ldots, n\right\} .
$$

We claim that $Z^{0, \kappa}(X)=A$. If this claim is true, it is clear that $Z^{0, \kappa}(X)=\mathbb{Z}$ and we find $H^{0, \kappa}(X)=\mathbb{Z}$.

Let us prove the claim. Choose two points $p_{r_{i}}, p_{s_{i}} \in X$. Since $X$ is $\kappa$-path connected, there is a path $\sigma_{i}$ in $X$ from $p_{r_{i}}$ to $p_{s_{i}}$ for each $i$. $\sigma_{i}$ is the set of digital 1 -simplexes that $\kappa$-path in $X$ from $p_{r_{i}}$ to $p_{s_{i}}$.

$$
\sigma_{i}=\left\{\left\langle p_{r_{i}}, p_{r+1_{i}}\right\rangle,\left\langle p_{r+1_{i}}, p_{r+2_{i}}\right\rangle, \ldots,\left\langle p_{s-1_{i}}, p_{s_{i}}\right\rangle\right\} .
$$

Let $e_{k_{i}}=\left\langle p_{k_{i}}, p_{k+1_{i}}\right\rangle$, for $k=r, r+1, \ldots, s$. We get

$$
\sigma_{i}=\left\{e_{r_{i}}, e_{r+1_{i}}, \ldots, e_{s-1_{i}}\right\}
$$

It is clear that

$$
\begin{gathered}
\partial_{1}\left(e_{r_{i}}\right)=p_{r+1_{i}}-p_{r_{i}}, \\
\partial_{1}\left(e_{r+1_{i}}\right)=p_{r+2_{i}}-p_{r+1_{i}}, \\
\partial_{1}\left(e_{r+2_{i}}\right)=p_{r+3_{i}}-p_{r+2_{i}}, \\
\vdots \\
\partial_{1}\left(e_{s-2_{i}}\right)=p_{s-1_{i}}-p_{s-2_{i}}, \\
\partial_{1}\left(e_{s-1_{i}}\right)=p_{s_{i}}-p_{s-1_{i}} .
\end{gathered}
$$

Hence we have

$$
\begin{aligned}
\delta^{0}\left(p_{r+1_{i}}\right) & =e_{r_{i}}-e_{r+1_{i}}, \\
\delta^{0}\left(p_{r+2_{i}}\right) & =e_{r+1_{i}}-e_{r+2_{i}}, \\
\vdots & \\
\delta^{0}\left(p_{s-1_{i}}\right) & =e_{s-2_{i}}-e_{s-1_{i}} .
\end{aligned}
$$

Let $\gamma_{i}$ is the set of digital 0 -simplexes on the path $\sigma_{i}$. For $\omega=\sum k \gamma_{i} \in Z$, we get

$$
\begin{aligned}
\delta^{0}(\omega) & =\delta^{0}\left(\sum k \gamma_{i}\right)=k \sum \delta^{0}\left(\gamma_{i}\right)=k \sum\left(e_{r_{i}}-e_{s-1_{i}}\right) \\
& =k \sum e_{r_{i}}-k \sum e_{s-1_{i}}=0 .
\end{aligned}
$$

So we get $\omega \in Z^{0, \kappa}(X)$.

Conversely, if $\theta \in Z^{0, \kappa}(X)$, then $\theta=\sum_{i=0}^{n} k_{i} p_{i} \in C^{0, \kappa}(X)$ and

$$
\delta^{0}(\theta)=\delta^{0}\left(\sum_{i=0}^{n} k_{i} p_{i}\right)=\sum_{i=0}^{n} k_{i} \delta^{0}\left(p_{i}\right)=0
$$

We have

$$
\begin{aligned}
\sum_{i=0}^{n} k_{i} \delta^{0}\left(\gamma_{i}\right) & =\sum_{i=0}^{n} k_{i}\left(e_{r_{i}}-e_{s-1_{i}}\right) \\
& =\sum_{i=0}^{n} k_{i} e_{r_{i}}-\sum_{i=0}^{n} k_{i} e_{s-1_{i}}=0 .
\end{aligned}
$$

Since $\sum k_{i} e_{r_{i}}=\sum k_{i} e_{s-1_{i}}, k_{i}=k$, for $i=0, \ldots, n$. Therefore we have that $\theta \in A$. Thus we find that $Z^{0, \kappa}(X)=A \cong \mathbb{Z}$ and

$$
H^{0, \kappa}(X)=Z^{0, \kappa}(X) / B^{0, \kappa}(X) \cong \mathbb{Z}
$$

Example 221 Let's compute the cohomology of $M S S_{18}^{\prime}$.

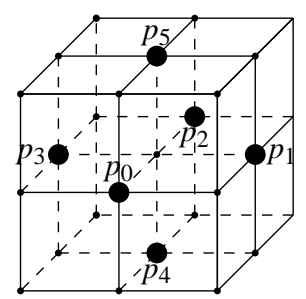

Fig. 6: $M S S_{18}^{\prime}$

$$
\text { Let }
$$$$
M S S_{18}^{\prime}=\left\{p_{0}=(1,1,0), p_{1}=(0,2,0), p_{2}=(-1,1,0),\right.
$$
$\left.p_{3}=(0,0,0), p_{4}=(0,1,-1), p_{5}=(0,1,1)\right\} \subset \mathbb{Z}^{3}$, where $p_{2}<p_{3}<p_{4}<p_{5}<p_{1}<p_{0}$.

$C_{0}^{18}\left(M_{S S}^{\prime}\right), C_{1}^{18}\left(M S S_{18}^{\prime}\right)$ and $C_{2}^{18}\left(M S S_{18}^{\prime}\right)$ are free abelian groups with bases, respectively,

O-simplexes

$$
\left\langle p_{0}\right\rangle,\left\langle p_{1}\right\rangle,\left\langle p_{2}\right\rangle,\left\langle p_{3}\right\rangle,\left\langle p_{4}\right\rangle,\left\langle p_{5}\right\rangle
$$

\section{1-simplexes}

$$
\begin{aligned}
& e_{0}=\left\langle p_{2} p_{1}\right\rangle, e_{1}=\left\langle p_{2} p_{3}\right\rangle, e_{2}=\left\langle p_{2} p_{4}\right\rangle, e_{3}=\left\langle p_{2} p_{5}\right\rangle, \\
& e_{4}=\left\langle p_{4} p_{1}\right\rangle, e_{5}=\left\langle p_{3} p_{4}\right\rangle, e_{6}=\left\langle p_{4} p_{0}\right\rangle, e_{7}=\left\langle p_{5} p_{1}\right\rangle, \\
& e_{8}=\left\langle p_{3} p_{5}\right\rangle, e_{9}=\left\langle p_{5} p_{0}\right\rangle, e_{10}=\left\langle p_{1} p_{0}\right\rangle, e_{11}=\left\langle p_{3} p_{0}\right\rangle \\
& \text { and }
\end{aligned}
$$

2-simplexes

$$
\begin{gathered}
\sigma_{0}=\left\langle p_{2} p_{4} p_{1}\right\rangle, \sigma_{1}=\left\langle p_{4} p_{1} p_{0}\right\rangle, \sigma_{2}=\left\langle p_{3} p_{4} p_{0}\right\rangle, \\
\sigma_{3}=\left\langle p_{2} p_{3} p_{4}\right\rangle, \sigma_{4}=\left\langle p_{2} p_{5} p_{1}\right\rangle, \sigma_{5}=\left\langle p_{2} p_{3} p_{5}\right\rangle, \\
\sigma_{6}=\left\langle p_{5} p_{1} p_{0}\right\rangle, \sigma_{7}=\left\langle p_{3} p_{5} p_{0}\right\rangle
\end{gathered}
$$

Since $C_{m}^{18}\left(M S S_{18}^{\prime}\right) \cong\{0\}$ for $m \geq 3$, we get the following short sequence: 
$0 \stackrel{\partial_{3}}{\longrightarrow} C_{2}^{18}\left(M S S_{18}^{\prime}\right) \stackrel{\partial_{2}}{\longrightarrow} C_{1}^{18}\left(M S S_{18}^{\prime}\right) \stackrel{\partial_{1}}{\longrightarrow} C_{0}^{18}\left(M S S_{18}^{\prime}\right) \stackrel{\partial_{0}}{\longrightarrow} 0$.

It is clear that

$$
\begin{aligned}
& C^{0,18}\left(M S S_{18}^{\prime}\right) \cong \operatorname{Hom}\left(C_{0}^{18}\left(M S S_{18}^{\prime}\right), \mathbb{Z}\right), \\
& C^{1,18}\left(M S S_{18}^{\prime}\right) \cong \operatorname{Hom}\left(C_{1}^{18}\left(M S S_{18}^{\prime}\right), \mathbb{Z}\right), \\
& C^{2,18}\left(M S S_{18}^{\prime}\right) \cong \operatorname{Hom}\left(C_{2}^{18}\left(M S S_{18}^{\prime}\right), \mathbb{Z}\right) .
\end{aligned}
$$

Hence we get the following sequence:

$$
\stackrel{\delta^{-1}}{\longrightarrow} C^{0,18}\left(M _ { S S _ { 1 8 } ^ { \prime } } ^ { \prime } \stackrel { \delta ^ { 0 } } { \longrightarrow } C ^ { 1 , 1 8 } \left(M_{S S_{18}^{\prime}}^{\prime} \stackrel{\delta^{1}}{\longrightarrow} C^{2,18}\left(M_{S S_{18}^{\prime}}\right) \stackrel{\delta^{2}}{\longrightarrow} 0 .\right.\right.
$$

By the definition, we obtain

$$
\begin{array}{ll}
\partial_{1}\left(e_{0}\right)=p_{1}-p_{2}, & \partial_{1}\left(e_{6}\right)=p_{0}-p_{4}, \\
\partial_{1}\left(e_{1}\right)=p_{3}-p_{2}, & \partial_{1}\left(e_{7}\right)=p_{1}-p_{5}, \\
\partial_{1}\left(e_{2}\right)=p_{4}-p_{2}, & \partial_{1}\left(e_{8}\right)=p_{5}-p_{3}, \\
\partial_{1}\left(e_{3}\right)=p_{5}-p_{2}, & \partial_{1}\left(e_{9}\right)=p_{0}-p_{5}, \\
\partial_{1}\left(e_{4}\right)=p_{1}-p_{4}, & \partial_{1}\left(e_{10}\right)=p_{0}-p_{1}, \\
\partial_{1}\left(e_{5}\right)=p_{4}-p_{3}, & \partial_{1}\left(e_{11}\right)=p_{0}-p_{3} .
\end{array}
$$

So we can find 0 -cochains,

$$
\begin{aligned}
& \delta^{0} p_{0}^{*}=e_{6}+e_{9}+e_{10}+e_{11}, \\
& \delta^{0} p_{1}^{*}=e_{0}+e_{4}+e_{7}-e_{10}, \\
& \delta^{0} p_{2}^{*}=-e_{0}-e_{1}-e_{2}-e_{3}, \\
& \delta^{0} p_{3}^{*}=e_{1}-e_{5}-e_{8}-e_{11}, \\
& \delta^{0} p_{4}^{*}=e_{2}-e_{4}+e_{5}-e_{6}, \\
& \delta^{0} p_{5}^{*}=e_{3}-e_{7}+e_{8}-e_{9} .
\end{aligned}
$$

From the definition of a homomorphism $\partial$, it is easy to see that

$$
\begin{aligned}
\partial_{2}\left(\sigma_{0}\right) & =\partial_{2}\left(\left\langle p_{2} p_{4} p_{1}\right\rangle\right)=\left\langle p_{4} p_{1}\right\rangle-\left\langle p_{2} p_{1}\right\rangle+\left\langle p_{2} p_{4}\right\rangle \\
& =e_{4}-e_{0}+e_{2}, \\
\partial_{2}\left(\sigma_{1}\right) & =\partial_{2}\left(\left\langle p_{4} p_{1} p_{0}\right\rangle\right)=\left\langle p_{1} p_{0}\right\rangle-\left\langle p_{4} p_{0}\right\rangle+\left\langle p_{4} p_{1}\right\rangle \\
& =e_{10}-e_{6}+e_{4}, \\
\partial_{2}\left(\sigma_{2}\right) & =\partial_{2}\left(\left\langle p_{3} p_{4} p_{0}\right\rangle\right)=\left\langle p_{4} p_{0}\right\rangle-\left\langle p_{3} p_{0}\right\rangle+\left\langle p_{3} p_{4}\right\rangle \\
& =e_{6}-e_{11}+e_{5}, \\
\partial_{2}\left(\sigma_{3}\right) & =\partial_{2}\left(\left\langle p_{2} p_{3} p_{4}\right\rangle\right)=\left\langle p_{3} p_{4}\right\rangle-\left\langle p_{2} p_{4}\right\rangle+\left\langle p_{2} p_{3}\right\rangle \\
& =e_{5}-e_{2}+e_{1}, \\
\partial_{2}\left(\sigma_{4}\right) & =\partial_{2}\left(\left\langle p_{2} p_{5} p_{1}\right\rangle\right)=\left\langle p_{5} p_{1}\right\rangle-\left\langle p_{2} p_{1}\right\rangle+\left\langle p_{2} p_{5}\right\rangle \\
& =e_{7}-e_{0}+e_{3}, \\
\partial_{2}\left(\sigma_{5}\right) & =\partial_{2}\left(\left\langle p_{2} p_{3} p_{5}\right\rangle\right)=\left\langle p_{3} p_{5}\right\rangle-\left\langle p_{2} p_{5}\right\rangle+\left\langle p_{2} p_{3}\right\rangle \\
& =e_{8}-e_{3}+e_{1}, \\
\partial_{2}\left(\sigma_{6}\right) & =\partial_{2}\left(\left\langle p_{5} p_{1} p_{0}\right\rangle\right)=\left\langle p_{1} p_{0}\right\rangle-\left\langle p_{5} p_{0}\right\rangle+\left\langle p_{5} p_{1}\right\rangle \\
& =e_{10}-e_{9}+e_{7}, \\
\partial_{2}\left(\sigma_{7}\right) & =\partial_{2}\left(\left\langle p_{3} p_{5} p_{0}\right\rangle\right)=\left\langle p_{5} p_{0}\right\rangle-\left\langle p_{3} p_{0}\right\rangle+\left\langle p_{3} p_{5}\right\rangle \\
& =e_{9}-e_{11}+e_{8} .
\end{aligned}
$$

Thus we can get 1-cochains,

$$
\begin{array}{lll}
\delta^{1} e_{0}^{*}=-\sigma_{0}-\sigma_{4}, & \delta^{1} e_{6}^{*}=-\sigma_{1}+\sigma_{2}, \\
\delta^{1} e_{1}^{*}=\sigma_{3}+\sigma_{5}, & \delta^{1} e_{7}^{*}=\sigma_{4}+\sigma_{6}, \\
\delta^{1} e_{2}^{*}=\sigma_{0}-\sigma_{3}, & \delta^{1} e_{8}^{*}=\sigma_{5}+\sigma_{7}, \\
\delta^{1} e_{3}^{*}=\sigma_{4}-\sigma_{5}, & & \delta^{1} e_{9}^{*}=-\sigma_{6}+\sigma_{7}, \\
\delta^{1} e_{4}^{*}=\sigma_{0}+\sigma_{1}, & & \delta^{1} e_{10}^{*}=\sigma_{1}+\sigma_{6}, \\
\delta^{1} e_{5}^{*}=\sigma_{2}+\sigma_{3}, & & \delta^{1} e_{11}^{*}=-\sigma_{2}-\sigma_{7} .
\end{array}
$$

We first find the kernel of $\delta^{0}$. We have

$$
\begin{aligned}
\delta^{0}\left(\sum_{i=0}^{5} n_{i} p_{i}^{*}\right)= & n_{0}\left(e_{6}+e_{9}+e_{10}+e_{11}\right) \\
& +n_{1}\left(e_{0}+e_{4}+e_{7}-e_{10}\right) \\
& +n_{2}\left(-e_{0}-e_{1}-e_{2}-e_{3}\right) \\
& +n_{3}\left(e_{1}-e_{5}-e_{8}-e_{11}\right) \\
& +n_{4}\left(e_{2}-e_{4}+e_{5}-e_{6}\right) \\
& +n_{5}\left(e_{3}-e_{7}+e_{8}-e_{9}\right) .
\end{aligned}
$$

Solving the equation

$$
\begin{aligned}
& e_{0}\left(n_{1}-n_{2}\right)+e_{1}\left(-n_{2}+n_{3}\right)+e_{2}\left(-n_{2}+n_{4}\right) \\
& +e_{3}\left(-n_{2}+n_{5}\right)+e_{4}\left(n_{1}-n_{4}\right)+e_{5}\left(-n_{3}+n_{4}\right) \\
& +e_{6}\left(n_{0}-n_{4}\right)+e_{7}\left(n_{1}-n_{5}\right)+e_{8}\left(-n_{3}+n_{5}\right) \\
& +e_{9}\left(-n_{5}+n_{0}\right)+e_{10}\left(n_{0}-n_{1}\right)+e_{11}\left(n_{0}-n_{3}\right)=0,
\end{aligned}
$$

we must have

$$
n_{0}=n_{1}=n_{2}=n_{3}=n_{4}=n_{5}=n .
$$

Hence, we get

$$
\begin{aligned}
Z^{0,18}\left(M_{18}^{\prime}\right) & =\left\{n\left(p_{0}+p_{1}+p_{2}+p_{3}+p_{4}+p_{5}\right) \mid n \in \mathbb{Z}\right\} \\
& \cong \mathbb{Z} .
\end{aligned}
$$

Since $B^{0,18}\left(M_{S S}^{\prime}\right) \cong 0$, the zero dimension cohomology group of $\mathrm{MSS}_{18}^{\prime}$ is isomorphic to the abelian group of integers.

Let

$$
\begin{aligned}
\delta^{1}\left(\sum_{i=0}^{11} k_{i} e_{i}^{*}\right)= & k_{0}\left(-\sigma_{0}-\sigma_{4}\right)+k_{1}\left(\sigma_{3}+\sigma_{5}\right)+k_{2}\left(\sigma_{0}-\sigma_{3}\right) \\
& +k_{3}\left(\sigma_{4}-\sigma_{5}\right)+k_{4}\left(\sigma_{0}+\sigma_{1}\right)+k_{5}\left(\sigma_{2}+\sigma_{3}\right) \\
& +k_{6}\left(-\sigma_{1}+\sigma_{2}\right)+k_{7}\left(\sigma_{4}+\sigma_{6}\right)+k_{8}\left(\sigma_{5}+\sigma_{7}\right) \\
& +k_{9}\left(-\sigma_{6}+\sigma_{7}\right)+k_{10}\left(\sigma_{1}+\sigma_{6}\right) \\
& +k_{11}\left(-\sigma_{2}-\sigma_{7}\right) .
\end{aligned}
$$

We find the kernel of $\delta^{1}$. We have the following equation

$$
\begin{aligned}
& \sigma_{0}\left(-k_{0}+k_{2}+k_{4}\right)+\sigma_{1}\left(k_{4}-k_{6}+k_{10}\right)+\sigma_{2}\left(k_{5}+k_{6}-k_{11}\right) \\
& \quad+\sigma_{3}\left(k_{1}-k_{2}+k_{5}\right)+\sigma_{4}\left(k_{3}+k_{7}-k_{0}\right)+\sigma_{5}\left(k_{1}+k_{8}-k_{3}\right) \\
& \quad+\sigma_{6}\left(k_{7}-k_{9}+k_{10}\right)+\sigma_{7}\left(k_{8}+k_{9}-k_{11}\right)=0 .
\end{aligned}
$$

Solving the equation above, we find

$$
\begin{aligned}
& k_{0}=k_{1}+k_{4}+k_{5}, \\
& k_{2}=k_{1}+k_{5}, \\
& k_{3}=k_{1}+k_{5}+k_{4}-k_{7}, \\
& k_{6}=k_{4}+k_{10}, \\
& k_{8}=k_{4}+k_{5}-k_{7}, \\
& k_{9}=k_{7}+k_{10}, \\
& k_{11}=k_{4}+k_{5}+k_{10} .
\end{aligned}
$$

Hence, we get the group of one dimensional cocycle

$Z^{1,18}\left(M_{S S}^{\prime}\right)=\left\{\left(k_{1}+k_{4}+k_{5}\right) e_{0}^{*}+k_{1} e_{1}^{*}+\left(k_{1}+k_{5}\right) e_{2}^{*}\right.$ $+\left(k_{1}+k_{4}+k_{5}-k_{7}\right) e_{3}^{*}+k_{4} e_{4}^{*}+k_{5} e_{5}^{*}$ 


$$
\begin{aligned}
& +\left(k_{4}+k_{10}\right) e_{6}^{*}+k_{7} e_{7}^{*}+\left(k_{4}+k_{5}-k_{7}\right) e_{8}^{*} \\
& +\left(k_{7}+k_{10}\right) e_{9}^{*}+k_{10} e_{10}^{*}+\left(k_{4}+k_{5}+k_{10}\right) e_{11}^{*} \\
& \left.\mid k_{i} \in \mathbb{Z}, i=1,4,5,7,10\right\} \cong \mathbb{Z}^{5} .
\end{aligned}
$$

On the other hand, we obtain the group of one dimensional coboundary

$$
\begin{aligned}
B^{1,18}\left(M S S_{18}^{\prime}\right)= & \left\{t_{0} e_{0}+t_{1} e_{1}+t_{2} e_{2}+t_{3} e_{3}+\left(t_{0}-t_{2}\right) e_{4}\right. \\
& +\left(t_{2}-t_{1}\right) e_{5}+t_{4} e_{6}+\left(t_{0}-t_{3}\right) e_{7} \\
& +\left(t_{3}-t_{1}\right) e_{8}+\left(t_{2}-t_{3}+t_{4}\right) e_{9} \\
& +\left(-t_{0}+t_{2}+t_{4}\right) e_{10}+\left(-t_{1}+t_{2}+t_{4}\right) e_{11} \\
& \left.\mid t_{i} \in \mathbb{Z}, i=0,1,2,3,4\right\} \cong \mathbb{Z}^{5} .
\end{aligned}
$$

Since $B^{1,18}\left(\operatorname{MSS}_{18}^{\prime}\right)=Z^{1,18}\left(M_{S S_{18}^{\prime}}^{\prime}\right)$, we have that,

$$
H^{1,18}\left(M S S_{18}^{\prime}\right) \cong\{0\}
$$

We find that

$$
\begin{aligned}
B^{2,18}\left(M S S_{18}^{\prime}\right)= & \left\{h_{0} \sigma_{0}+h_{1} \sigma_{1}+h_{2} \sigma_{2}+h_{3} \sigma_{3}+h_{4} \sigma_{4}\right. \\
& +h_{5} \sigma_{5}+h_{6} \sigma_{6} \\
& +\left(h_{5}-h_{6}+h_{4}-h_{0}-h_{3}+h_{2}+h_{1}\right) \sigma_{7} \\
& \left.\mid h_{i} \in \mathbb{Z}, i=0,1,2,3,4,5,6\right\} \cong \mathbb{Z}^{7}
\end{aligned}
$$

Since $Z^{2,18}\left(M S S_{18}^{\prime}\right) \cong \mathbb{Z}^{8}$, we have that,

$$
H^{2,18}\left(M S S_{18}^{\prime}\right) \cong \mathbb{Z} \text {. }
$$

Therefore we conclude that

$$
H^{q, 18}\left(M_{S S}^{\prime}{ }_{18}\right)=\left\{\begin{array}{l}
\mathbb{Z}, q=0,2 \\
0, q \neq 0,2
\end{array}\right.
$$

Example 222 Let's compute the cohomology of $M S S_{18}$.

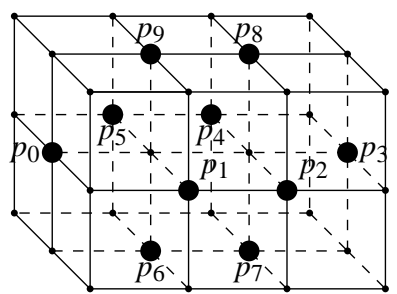

Fig. 7: $M S S_{18}$

Let $\mathrm{MSS}_{18}=\left\{p_{0}=(0,0,1), p_{1}=(1,1,1)\right.$, $p_{2}=(1,2,1), p_{3}=(0,3,1), p_{4}=(-1,2,1)$, $p_{5}=(-1,1,1), p_{6}=(0,1,0), p_{7}=(0,2,0)$, $\left.p_{8}=(0,2,2), p_{9}=(0,1,2)\right\} \subset \mathbb{Z}^{3}$, where $p_{5}<p_{4}<p_{0}<p_{6}<p_{9}<p_{7}<p_{8}<p_{3}<p_{1}<p_{2}$.

$C_{0}^{18}\left(M S S_{18}\right), C_{1}^{18}\left(M S S_{18}\right)$ and $C_{2}^{18}\left(M S S_{18}\right)$ are free abelian groups with bases, respectively,

\section{O-simplexes}

$\left\langle p_{0}\right\rangle,\left\langle p_{1}\right\rangle,\left\langle p_{2}\right\rangle,\left\langle p_{3}\right\rangle,\left\langle p_{4}\right\rangle,\left\langle p_{5}\right\rangle,\left\langle p_{6}\right\rangle,\left\langle p_{7}\right\rangle,\left\langle p_{8}\right\rangle,\left\langle p_{9}\right\rangle$

1-simplexes

$$
\begin{gathered}
e_{0}=\left\langle p_{0} p_{1}\right\rangle, e_{1}=\left\langle p_{0} p_{9}\right\rangle, e_{2}=\left\langle p_{5} p_{0}\right\rangle, e_{3}=\left\langle p_{0} p_{6}\right\rangle, \\
e_{4}=\left\langle p_{9} p_{8}\right\rangle, e_{5}=\left\langle p_{9} p_{1}\right\rangle, e_{6}=\left\langle p_{5} p_{9}\right\rangle, e_{7}=\left\langle p_{6} p_{1}\right\rangle, \\
e_{8}=\left\langle p_{1} p_{2}\right\rangle, e_{9}=\left\langle p_{5} p_{6}\right\rangle, e_{10}=\left\langle p_{6} p_{7}\right\rangle, e_{11}=\left\langle p_{5} p_{4}\right\rangle, \\
e_{12}=\left\langle p_{8} p_{2}\right\rangle, e_{13}=\left\langle p_{4} p_{8}\right\rangle, e_{14}=\left\langle p_{8} p_{3}\right\rangle, e_{15}=\left\langle p_{4} p_{3}\right\rangle, \\
e_{16}=\left\langle p_{4} p_{7}\right\rangle, e_{17}=\left\langle p_{3} p_{2}\right\rangle, e_{18}=\left\langle p_{7} p_{2}\right\rangle, e_{19}=\left\langle p_{7} p_{3}\right\rangle \\
\quad \text { and }
\end{gathered}
$$

2-simplexes

$$
\begin{gathered}
\sigma_{0}=\left\langle p_{0} p_{9} p_{1}\right\rangle, \sigma_{1}=\left\langle p_{0} p_{6} p_{1}\right\rangle, \sigma_{2}=\left\langle p_{5} p_{0} p_{6}\right\rangle, \\
\sigma_{3}=\left\langle p_{5} p_{0} p_{9}\right\rangle, \sigma_{4}=\left\langle p_{4} p_{8} p_{3}\right\rangle, \sigma_{5}=\left\langle p_{4} p_{7} p_{3}\right\rangle, \\
\sigma_{6}=\left\langle p_{8} p_{3} p_{2}\right\rangle, \sigma_{7}=\left\langle p_{7} p_{3} p_{2}\right\rangle
\end{gathered}
$$

Since $C_{m}^{18}\left(M S S_{18}\right)$ is a trivial group for $m \geq 3$, we have

$$
0 \stackrel{\partial_{3}}{\longrightarrow} C_{2}^{18}\left(M S S_{18}\right) \stackrel{\partial_{2}}{\longrightarrow} C_{1}^{18}\left(M S S_{18}\right) \stackrel{\partial_{1}}{\longrightarrow} C_{0}^{18}\left(M S S_{18}\right) \stackrel{\partial_{0}}{\longrightarrow} 0 .
$$

By the definition of cochain, we obtain

$$
\begin{aligned}
& C^{0,18}\left(M_{S S}\right) \cong \operatorname{Hom}\left(C_{0}^{18}\left(M_{18} S_{18}\right), \mathbb{Z}\right), \\
& C^{1,18}\left(M_{18}\right) \cong \operatorname{Hom}\left(C_{1}^{18}\left(M_{18}\right), \mathbb{Z}\right), \\
& C^{2,18}\left(M_{18}\right) \cong \operatorname{Hom}\left(C_{2}^{18}\left(M S S_{18}\right), \mathbb{Z}\right) .
\end{aligned}
$$

Hence we get

$$
\stackrel{\delta^{-1}}{\longrightarrow} C^{0,18}\left(M_{S S} S_{18}\right) \stackrel{\delta^{0}}{\longrightarrow} C^{1,18}\left(M_{S S}\right) \stackrel{\delta^{1}}{\longrightarrow} C^{2,18}\left(M_{S S} S_{18}\right) \stackrel{\delta^{2}}{\longrightarrow} 0
$$

It is easy to see that

$$
\begin{array}{ll}
\partial_{1}\left(e_{0}\right)=p_{1}-p_{0}, & \partial_{1}\left(e_{10}\right)=p_{7}-p_{6}, \\
\partial_{1}\left(e_{1}\right)=p_{9}-p_{0}, & \partial_{1}\left(e_{11}\right)=p_{4}-p_{5}, \\
\partial_{1}\left(e_{2}\right)=p_{0}-p_{5}, & \partial_{1}\left(e_{12}\right)=p_{2}-p_{8}, \\
\partial_{1}\left(e_{3}\right)=p_{6}-p_{0}, & \partial_{1}\left(e_{13}\right)=p_{8}-p_{4}, \\
\partial_{1}\left(e_{4}\right)=p_{8}-p_{9}, & \partial_{1}\left(e_{14}\right)=p_{3}-p_{8}, \\
\partial_{1}\left(e_{5}\right)=p_{1}-p_{9}, & \partial_{1}\left(e_{15}\right)=p_{3}-p_{4}, \\
\partial_{1}\left(e_{6}\right)=p_{9}-p_{5}, & \partial_{1}\left(e_{16}\right)=p_{7}-p_{4}, \\
\partial_{1}\left(e_{7}\right)=p_{1}-p_{6}, & \partial_{1}\left(e_{17}\right)=p_{2}-p_{3}, \\
\partial_{1}\left(e_{8}\right)=p_{2}-p_{1}, & \partial_{1}\left(e_{18}\right)=p_{2}-p_{7}, \\
\partial_{1}\left(e_{9}\right)=p_{6}-p_{5}, & \partial_{1}\left(e_{19}\right)=p_{3}-p_{7} .
\end{array}
$$

So we find 0-cochains,

$$
\begin{aligned}
& \delta^{0} p_{0}^{*}=-e_{0}-e_{1}+e_{2}-e_{3}, \\
& \delta^{0} p_{1}^{*}=e_{0}+e_{5}+e_{7}-e_{8}, \\
& \delta^{0} p_{2}^{*}=e_{8}+e_{12}+e_{17}+e_{18}, \\
& \delta^{0} p_{3}^{*}=e_{14}+e_{15}-e_{17}+e_{19}, \\
& \delta^{0} p_{4}^{*}=e_{11}-e_{13}-e_{15}-e_{16}, \\
& \delta^{0} p_{5}^{*}=-e_{2}-e_{6}-e_{9}-e_{11}, \\
& \delta^{0} p_{6}^{*}=e_{3}-e_{7}+e_{9}-e_{10}, \\
& \delta^{0} p_{7}^{*}=e_{10}+e_{16}-e_{18}-e_{19}, \\
& \delta^{0} p_{8}^{*}=e_{4}-e_{12}+e_{13}-e_{14}, \\
& \delta^{0} p_{9}^{*}=e_{1}-e_{4}-e_{5}+e_{6} .
\end{aligned}
$$


From the definition, we can easily obtain

$$
\begin{aligned}
& \partial_{2}\left(\sigma_{0}\right)=\left\langle p_{9} p_{1}\right\rangle-\left\langle p_{0} p_{1}\right\rangle+\left\langle p_{0} p_{9}\right\rangle=e_{5}-e_{0}+e_{1}, \\
& \partial_{2}\left(\sigma_{1}\right)=\left\langle p_{6} p_{1}\right\rangle-\left\langle p_{0} p_{1}\right\rangle+\left\langle p_{0} p_{6}\right\rangle=e_{7}-e_{0}+e_{3}, \\
& \partial_{2}\left(\sigma_{2}\right)=\left\langle p_{0} p_{6}\right\rangle-\left\langle p_{5} p_{6}\right\rangle+\left\langle p_{5} p_{0}\right\rangle=e_{3}-e_{9}+e_{2}, \\
& \partial_{2}\left(\sigma_{3}\right)=\left\langle p_{0} p_{9}\right\rangle-\left\langle p_{5} p_{9}\right\rangle+\left\langle p_{5} p_{0}\right\rangle=e_{1}-e_{6}+e_{2}, \\
& \partial_{2}\left(\sigma_{4}\right)=\left\langle p_{8} p_{3}\right\rangle-\left\langle p_{4} p_{3}\right\rangle+\left\langle p_{4} p_{8}\right\rangle=e_{14}-e_{15}+e_{13}, \\
& \partial_{2}\left(\sigma_{5}\right)=\left\langle p_{7} p_{3}\right\rangle-\left\langle p_{4} p_{3}\right\rangle+\left\langle p_{4} p_{7}\right\rangle=e_{19}-e_{15}+e_{16}, \\
& \partial_{2}\left(\sigma_{6}\right)=\left\langle p_{3} p_{2}\right\rangle-\left\langle p_{8} p_{2}\right\rangle+\left\langle p_{8} p_{3}\right\rangle=e_{17}-e_{12}+e_{14}, \\
& \partial_{2}\left(\sigma_{7}\right)=\left\langle p_{3} p_{2}\right\rangle-\left\langle p_{7} p_{2}\right\rangle+\left\langle p_{7} p_{3}\right\rangle=e_{17}-e_{18}+e_{19} .
\end{aligned}
$$

Thus, we get 1-cochains,

$$
\begin{array}{ll}
\delta^{1} e_{0}^{*}=-\sigma_{0}-\sigma_{1}, & \delta^{1} e_{10}^{*}=\{0\}, \\
\delta^{1} e_{1}^{*}=\sigma_{0}+\sigma_{3}, & \delta^{1} e_{11}^{*}=\{0\}, \\
\delta^{1} e_{2}^{*}=\sigma_{2}+\sigma_{3}, & \delta^{1} e_{12}^{*}=-\sigma_{6}, \\
\delta^{1} e_{3}^{*}=\sigma_{1}+\sigma_{2}, & \delta^{1} e_{13}^{*}=\sigma_{4}, \\
\delta^{1} e_{4}^{*}=\{0\}, & \delta^{1} e_{14}^{*}=\sigma_{4}+\sigma_{6}, \\
\delta^{1} e_{5}^{*}=\sigma_{0}, & \delta^{1} e_{15}^{*}=-\sigma_{4}-\sigma_{5}, \\
\delta^{1} e_{6}^{*}=-\sigma_{3}, & \delta^{1} e_{16}^{*}=\sigma_{5}, \\
\delta^{1} e_{7}^{*}=\sigma_{1}, & \delta^{1} e_{17}^{*}=\sigma_{6}+\sigma_{7}, \\
\delta^{1} e_{8}^{*}=\{0\}, & \delta^{1} e_{18}^{*}=-\sigma_{7}, \\
\delta^{1} e_{9}^{*}=-\sigma_{2}, & \delta^{1} e_{19}^{*}=\sigma_{5}+\sigma_{7} .
\end{array}
$$

Let's find the kernel of $\delta^{0}$. By the definition of $\delta^{0}$, we see that

$$
\begin{aligned}
\delta^{0}\left(\sum_{i=0}^{9} n_{i} p_{i}^{*}\right)=n_{0}( & \left.-e_{0}-e_{1}+e_{2}-e_{3}\right) \\
& +n_{1}\left(e_{0}+e_{5}+e_{7}-e_{8}\right) \\
& +n_{2}\left(e_{8}+e_{12}+e_{17}+e_{18}\right) \\
& +n_{3}\left(e_{14}+e_{15}-e_{17}+e_{19}\right) \\
& +n_{4}\left(e_{11}-e_{13}-e_{15}-e_{16}\right) \\
& +n_{5}\left(-e_{2}-e_{6}-e_{9}-e_{11}\right) \\
& +n_{6}\left(e_{3}-e_{7}+e_{9}-e_{10}\right) \\
& +n_{7}\left(e_{10}+e_{16}-e_{18}-e_{19}\right) \\
& +n_{8}\left(e_{4}-e_{12}+e_{13}-e_{14}\right) \\
& +n_{9}\left(e_{1}-e_{4}-e_{5}+e_{6}\right) .
\end{aligned}
$$

Solving the equation

$$
\begin{aligned}
& e_{0}\left(-n_{0}+n_{1}\right)+e_{1}\left(-n_{0}+n_{9}\right)+e_{2}\left(n_{0}-n_{5}\right) \\
& \quad+e_{3}\left(-n_{0}+n_{6}\right)+e_{4}\left(n_{8}-n_{9}\right)+e_{5}\left(n_{1}-n_{9}\right) \\
& \quad+e_{6}\left(-n_{5}+n_{9}\right)+e_{7}\left(n_{1}-n_{6}\right)+e_{8}\left(n_{2}-n_{1}\right) \\
& \quad+e_{9}\left(-n_{5}+n_{6}\right)+e_{10}\left(-n_{6}+n_{7}\right)+e_{11}\left(n_{4}-n_{5}\right) \\
& \quad+e_{12}\left(n_{2}-n_{8}\right)+e_{13}\left(-n_{4}+n_{8}\right)+e_{14}\left(n_{3}-n_{8}\right) \\
& \quad+e_{15}\left(n_{3}-n_{4}\right)+e_{16}\left(-n_{4}+n_{7}\right)+e_{17}\left(n_{2}-n_{3}\right) \\
& \quad+e_{18}\left(n_{2}-n_{7}\right)+e_{19}\left(n_{3}-n_{7}\right)=0,
\end{aligned}
$$

we find

$$
n_{0}=n_{1}=n_{2}=n_{3}=n_{4}=n_{5}=n_{6}=n_{7}=n_{8}=n_{9}=n .
$$

Hence, we get the group of zero dimensional cocycles

$$
\begin{aligned}
Z^{0,18}\left(M S S_{18}\right)=\{ & n\left(p_{0}+p_{1}+p_{2}+p_{3}+p_{4}+p_{5}\right. \\
& \left.\left.+p_{6}+p_{7}+p_{8}+p_{9}\right) \mid n \in \mathbb{Z}\right\} \cong \mathbb{Z} .
\end{aligned}
$$

Since $B^{0,18}\left(M_{S S}\right) \cong 0$, we obtain

$$
H^{0,18}\left(M S S_{18}\right) \cong \mathbb{Z}
$$

Let

$$
\begin{aligned}
\delta^{1}\left(\sum_{i=0}^{19} k_{i} e_{i}^{*}\right)= & k_{0}\left(-\sigma_{0}-\sigma_{1}\right)+k_{1}\left(\sigma_{0}+\sigma_{3}\right)+k_{2}\left(\sigma_{2}+\sigma_{3}\right) \\
& +k_{3}\left(\sigma_{1}+\sigma_{2}\right)+k_{4}(\{0\})+k_{5}\left(\sigma_{0}\right) \\
& +k_{6}\left(-\sigma_{3}\right)+k_{7}\left(\sigma_{1}\right)+k_{8}(\{0\}) \\
& +k_{9}\left(-\sigma_{2}\right)+k_{10}(\{0\})+k_{11}(\{0\}) \\
& +k_{12}\left(-\sigma_{6}\right)+k_{13}\left(\sigma_{4}\right)+k_{14}\left(\sigma_{4}+\sigma_{6}\right) \\
& +k_{15}\left(-\sigma_{4}-\sigma_{5}\right)+k_{16}\left(\sigma_{5}\right) \\
& +k_{17}\left(\sigma_{6}+\sigma_{7}\right)+k_{18}\left(-\sigma_{7}\right)+k_{19}\left(\sigma_{5}+\sigma_{7}\right) .
\end{aligned}
$$

We find the kernel of $\delta^{1}$. We have

$$
\begin{aligned}
& \sigma_{0}\left(-k_{0}+k_{1}+k_{5}\right)+\sigma_{1}\left(-k_{0}+k_{3}+k_{7}\right) \\
& +\sigma_{2}\left(k_{2}+k_{3}-k_{9}\right)+\sigma_{3}\left(k_{1}+k_{2}-k_{6}\right) \\
& +\sigma_{4}\left(k_{13}+k_{14}-k_{15}\right)+\sigma_{5}\left(-k_{15}+k_{16}+k_{19}\right) \\
& +\sigma_{6}\left(-k_{12}+k_{14}+k_{17}\right)+\sigma_{7}\left(k_{17}-k_{18}+k_{19}\right)=0 .
\end{aligned}
$$

Solving the equation above, we get

$$
\begin{aligned}
& k_{0}=k_{1}+k_{5}, \\
& k_{6}=k_{1}+k_{2}, \\
& k_{7}=k_{1}+k_{5}-k_{3}, \\
& k_{9}=k_{2}+k_{3}, \\
& k_{12}=k_{14}+k_{17}, \\
& k_{15}=k_{13}+k_{14}, \\
& k_{18}=k_{13}+k_{14}-k_{16}+k_{17}, \\
& k_{19}=k_{13}+k_{14}-k_{16} .
\end{aligned}
$$

Hence, we obtain

$$
\begin{aligned}
Z^{1,18}\left(M S S_{18}\right)=\{( & \left.k_{1}+k_{5}\right) e_{0}^{*}+k_{1} e_{1}^{*}+k_{2} e_{2}^{*}+k_{3} e_{3}^{*} \\
& +k_{4} e_{4}^{*}+k_{5} e_{5}^{*}+\left(k_{1}+k_{2}\right) e_{6}^{*} \\
& +\left(k_{1}+k_{5}-k_{3}\right) e_{7}^{*}+k_{8} e_{8}^{*} \\
& +\left(k_{2}+k_{3}\right) e_{9}^{*}+k_{10} e_{10}^{*}+k_{11} e_{11}^{*} \\
& +\left(k_{14}+k_{17}\right) e_{12}^{*}+k_{13} e_{13}^{*}+k_{14} e_{14}^{*} \\
& +\left(k_{13}+k_{14}\right) e_{15}^{*}+k_{16} e_{16}^{*} \\
& +k_{17} e_{17}^{*}+\left(k_{13}+k_{14}-k_{16}+k_{17}\right) e_{18}^{*} \\
& +\left(k_{13}+k_{14}-k_{16}\right) e_{19}^{*} \\
& \left.\mid k_{i} \in \mathbb{Z}, i=1,4,5,7,10\right\} \cong \mathbb{Z}^{12} .
\end{aligned}
$$

On the other hand, we obtain

$$
\begin{aligned}
& B^{1,18}\left(M S S_{18}\right)=\left\{t_{0} e_{0}+t_{1} e_{1}+t_{2} e_{2}+t_{3} e_{3}+t_{4} e_{4}\right. \\
&+\left(t_{0}-t_{1}\right) e_{5}+\left(t_{1}+t_{2}\right) e_{6}+\left(t_{0}-t_{3}\right) e_{7} \\
&+t_{5} e_{8}+\left(t_{2}+t_{3}\right) e_{9}+t_{6} e_{10}+t_{7} e_{11} \\
&+\left(t_{0}-t_{1}-t_{4}+t_{5}\right) e_{12}+\left(t_{1}+t_{2}+t_{4}-t_{7}\right) e_{13} \\
&+t_{8} e_{14}+\left(t_{1}+t_{2}+t_{4}-t_{7}+t_{8}\right) e_{15} \\
&+\left(t_{2}+t_{3}+t_{6}-t_{7}\right) e_{16}+\left(t_{0}-t_{1}-t_{4}+t_{5}-t_{8}\right) e_{17} \\
&+\left(t_{0}-t_{3}+t_{5}-t_{6}\right) e_{18}+\left(t_{1}-t_{3}+t_{4}-t_{6}+t_{8}\right) e_{19} \\
&\left.\mid t_{i} \in \mathbb{Z}, i=0,1,2,3,4,5,6,7,8\right\} \cong \mathbb{Z}^{9} .
\end{aligned}
$$

We have that,

$$
H^{1,18}\left(M S S_{18}\right) \cong \mathbb{Z}^{3} .
$$


We find that

$$
\begin{aligned}
B^{2,18}\left(M S S_{18}\right)=\{ & h_{0} \sigma_{0}+h_{1} \sigma_{1}+h_{2} \sigma_{2}+h_{3} \sigma_{3}+h_{4} \sigma_{4} \\
& +h_{5} \sigma_{5}+h_{6} \sigma_{6}+h_{7} \sigma_{7} \\
& \left.\mid h_{i} \in \mathbb{Z}, i=0,1,2,3,4,5,6,7\right\} \cong \mathbb{Z}^{8} .
\end{aligned}
$$

Since $Z^{2,18}\left(M_{S S}\right) \cong \mathbb{Z}^{8}$, we have that,

$$
H^{2,18}\left(M_{18}\right) \cong\{0\} .
$$

We get our result

$$
H^{q, 18}\left(M_{18}\right)=\left\{\begin{array}{c}
\mathbb{Z}, q=0 \\
\mathbb{Z}^{3}, q=1 \\
0, q \geq 2
\end{array}\right.
$$

\section{Digital Relative Cohomology Groups}

Definition 31[22] Let $(K, \kappa) \subset \mathbb{Z}^{n}$ be a digital simplicial complex and $\left(K_{0}, \kappa\right)$ be a digital subcomplex of $(K, \kappa)$. For any abelian group $G$ and for any $p \in \mathbb{Z}$, the p-dimensional digital relative cochain group

$$
C^{p, \kappa}\left(K, K_{0} ; G\right)=\operatorname{Hom}\left(C_{p}^{\kappa}\left(K, K_{0}\right), G\right)
$$

The boundary operator

$$
\delta^{p}: C^{p, \kappa}\left(K, K_{0} ; G\right) \rightarrow C^{p+1, \kappa}\left(K, K_{0} ; G\right)
$$

is the dual homomorphism of $\partial_{p+1}$.

$Z^{p, \kappa}\left(K, K_{0} ; G\right)$ is kernel of this homomorphism, $B^{p+1, \kappa}\left(K, K_{0} ; G\right)$ is image of its. These are called the group of digital relative simlicial $p$-cocycles and the group of digital relative simlicial $p$-coboundaries, respectively. Then the $p$ th digital relative simlicial cohomology group of $\left(K, K_{0}\right)$ is the quotient group

$$
H^{p, \kappa}\left(K, K_{0} ; G\right)=Z^{p, \kappa}\left(K, K_{0} ; G\right) / B^{p, \kappa}\left(K, K_{0} ; G\right)
$$

The qutient group $C^{p, \kappa}(K ; G) / C^{p, \kappa}\left(K_{0} ; G\right)$ is called the group of relative chains of $K$ modulo $K_{0}$ and is denoted by $C^{p, \kappa}\left(K, K_{0} ; G\right) . C^{p, \kappa}\left(K, K_{0} ; G\right)$ is subgroup of $C^{p, \kappa}(K ; G)$.

A digital relative cochain $C^{p, \kappa}\left(K, K_{0} ; G\right)$ is a homomorphism from $C_{p}^{\kappa}\left(K, K_{0}\right)$ to $G$. The group of such homomorphisms corresponds precisely to the group of all homomorphisms of $C_{p}^{\kappa}(K)$ into $G$ that vanish on the subgroup $C_{p}^{\kappa}\left(K_{0}\right)$. This is just a subgroup of the group of all homomorphisms of $C_{p}^{\kappa}(K)$ into $G$. Thus $C^{p, \kappa}\left(K, K_{0} ; G\right)$ can be naturally considered to be the subgroup of $C^{p, \kappa}(K ; G)$ consisting of those cochains that vanish on every simplex of $K_{0}$.

For chains, we had an exact sequence

$$
0 \longrightarrow C_{p}^{\kappa}\left(K_{0}\right) \stackrel{i}{\longrightarrow} C_{p}^{\kappa}(K) \stackrel{j}{\longrightarrow} C_{p}^{\kappa}\left(K, K_{0}\right) \longrightarrow 0
$$

where $C_{p}^{\mathcal{K}}\left(K_{0}\right)$ is a subgroup of $C_{p}^{\mathcal{K}}(K)$, and $C_{p}^{\mathcal{K}}\left(K, K_{0}\right)$ is their quotient. The sequence splits because the relative chain group is free. Therefore, the sequence

$$
0 \longleftarrow C^{p, \kappa}\left(K_{0} ; G\right) \stackrel{\widetilde{i}}{\longleftarrow} C^{p, \kappa}(K ; G) \stackrel{\widetilde{j}}{\longleftarrow} C^{p, \kappa}\left(K, K_{0} ; G\right) \longleftarrow 0
$$

is exact and splits. The dual of the projection map $j$ is an inclusion map $\tilde{j}$ and the dual of the inclusion map $i$ is a restriction map $\tilde{i}$.

Let us now consider the homomorphism of cohomology induced by a digital simplicial map. Recall that if

$$
f:\left(K, K_{0}\right) \rightarrow\left(L, L_{0}\right)
$$

is a simplicial map, then one has a corresponding chain map

$$
f_{\sharp}: C_{p, \kappa}\left(K, K_{0}\right) \rightarrow C_{p, \kappa}\left(L, L_{0}\right)
$$

The dual of $f_{\sharp}$ maps cochains to cochains; we usually denote it by $f^{\sharp}$. Because $f_{\sharp}$ commutes with $\partial$, the map $f^{\sharp}$ commutes with $\delta$, since the dual of the equation $f_{\sharp} \circ \partial=\partial \circ f_{\sharp}$ is the equation $\delta \circ f^{\sharp}=f^{\sharp} \circ \delta$. Hence $f^{\sharp}$ carries cocycles to cocycles and coboundaries to coboundaries. $f^{\sharp}$ is called a cochain map; it induces a homomorphism of digital cohomology groups,

$$
H^{p, \kappa}\left(K, K_{0} ; G\right) \stackrel{f^{*}}{\longleftarrow} H^{p, \kappa}\left(L, L_{0} ; G\right)
$$

Functoriality holds, even on the cochain level. For if $i$ is the identity, then $i_{\sharp}$ is the identity and so is $i^{\sharp}$. Similarly, the equation $(g \circ f)_{\sharp}=g_{\sharp} \circ f_{\sharp}$ gives, when dualized, the equation $(g \circ f)^{\sharp}=f^{\sharp} \circ g^{\sharp}$.

Just as in the case of homology, one has a long exact sequence in cohomology involving the relative groups. But again, there are a few differences.

Theorem 32[22] Let $K_{0}$ be a subcomplex of a digital complex $K$. There exists a long exact sequence

$$
\begin{gathered}
\ldots \leftarrow H^{q, \kappa}\left(K_{0} ; G\right) \leftarrow H^{q, \kappa}(K ; G) \leftarrow H^{q, \kappa}\left(K, K_{0} ; G\right) \leftarrow \\
H^{q-1, \kappa}\left(K_{0} ; G\right) \leftarrow \ldots
\end{gathered}
$$

Proof. If we apply the Zig-zag Lemma to the diagram

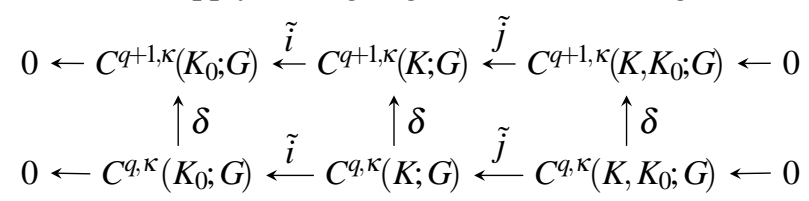

this theorem follows.

Example 33 We consider $A=\{(0,1,1)\}$ as subspace of $M S S_{18}^{\prime}=\left\{p_{0}=(1,1,0), p_{1}=(0,2,0), p_{2}=(-1,1,0)\right.$, 


$$
\left.p_{3}=(0,0,0), p_{4}=(0,1,-1), p_{5}=(0,1,1)\right\} \subset \mathbb{Z}^{3}
$$

Let us compute $H^{q, 18}\left(\mathrm{MSS}_{18}^{\prime}, A\right)$ with Theorem 32. Since $A$ is a single vertex, from Theorem 2.19 we conclude that

$$
H^{q, 18}(A)=\left\{\begin{array}{l}
\mathbb{Z}, q=0 \\
0, q \neq 0 .
\end{array}\right.
$$

From Example 221, we get

$$
H^{q, 18}\left(M S S_{18}^{\prime}\right)=\left\{\begin{array}{l}
\mathbb{Z}, q=0,2 \\
0, q \neq 0,2
\end{array}\right.
$$

By Theorem 32, we have the exact sequence

$$
\begin{gathered}
\ldots \rightarrow H^{q, 18}\left(M_{\left.S S_{18}^{\prime}, A\right) \rightarrow H^{q, 18}}^{\prime}\left(M_{S S_{18}^{\prime}}^{\prime}\right) \rightarrow H^{q, 18}(A) \rightarrow\right. \\
H^{q+1,18}\left(M^{\prime} S_{18}^{\prime}, A\right) \rightarrow \ldots
\end{gathered}
$$

We get $H^{0,18}\left(M_{S S_{18}^{\prime}}^{\prime} A\right) \cong \mathbb{Z}$, from Theorem 219 .

$$
0 \stackrel{\delta^{0}}{\longrightarrow} \mathbb{Z} \stackrel{j^{*}}{\longrightarrow} \mathbb{Z} \stackrel{i^{*}}{\longrightarrow} \mathbb{Z} \stackrel{\delta^{1}}{\longrightarrow} H^{1,18}\left(M_{S S}^{\prime}{ }_{18}, A\right) \stackrel{k^{*}}{\longrightarrow} 0
$$

Applying to the First Isomorphism Theorem, we have

$$
H^{1,18}\left(\operatorname{MSS}_{18}^{\prime}, A\right) / \operatorname{Ker} k^{*} \cong \operatorname{Im} k^{*} .
$$

Since the sequence is exact, we have $\operatorname{Im} \delta^{1}=\operatorname{Ker} k^{*}$ and $\operatorname{Im} i^{*}=\operatorname{Ker} \delta^{1}$. As $i^{*}$ is isomorphism, we conclude that Ker $i^{*}=0$ and $\operatorname{Im} i^{*}=\mathbb{Z}$. We find $\operatorname{Ker} \delta^{1}=\mathbb{Z}$. Again applying the First Isomorphism Theorem, since

$$
\mathbb{Z} / \operatorname{Ker} \delta^{1} \cong \operatorname{Im} \delta^{1}
$$

we have $\operatorname{Im} \delta^{1}=0=\operatorname{Ker} k^{*}$ and

$$
H^{1,18}\left(M_{S S}^{\prime}, A\right)=0 \text {. }
$$

Therefore we get

$$
H^{q, 18}\left(M_{S S}^{\prime}, A\right)=\left\{\begin{array}{l}
\mathbb{Z}, q=0 \\
0, q \neq 0 .
\end{array}\right.
$$

\section{Cup Product For Digital Images}

Definition 41[23] Let $(X, \kappa)$ be a digital simplicial complex. Suppose that the coefficient group $G$ is the additive group of a commutative ring with identity. The digital simplicial cup product

$$
\smile: C^{p, \kappa}(X, G) \times C^{q, \kappa}(X, G) \rightarrow C^{p+q, \kappa}(X, G)
$$

of cochains $c^{p}$ and $c^{q}$ is defined by the formula $<c^{p} \smile c^{q},\left[v_{0}, \ldots, v_{p+q}\right]>=<c^{p},\left[v_{0}, \ldots, v_{p}\right]>$ $.<c^{q},\left[v_{p}, \ldots, v_{p+q}\right]>$

where $v_{0}<\ldots<v_{p+q}$ in the given ordering and '.' is the product in $G$.

Theorem 42[23] Let $\alpha, \alpha_{1}, \alpha_{2} \in H^{p, \kappa}\left(X, G_{1}\right)$ and $\beta, \beta_{1}, \beta_{2} \in H^{q, \kappa}\left(X, G_{2}\right)$, we get

$$
\left(\alpha_{1}+\alpha_{2}\right) \smile \beta=\alpha_{1} \smile \beta+\alpha_{2} \smile \beta
$$

and

$$
\alpha \smile\left(\beta_{1}+\beta_{2}\right)=\alpha \smile \beta_{1}+\alpha \smile \beta_{2} .
$$

Theorem 43[23]

$\delta\left(c^{p} \smile c^{q}\right)=\delta c^{p} \smile c^{q}+(-1)^{p} c^{p} \smile \delta c^{q}$.

Theorem 44[23] Let $(X, \kappa)$ be a digital simplicial complex. The cup product on digital simplicial cochains is associative, that is,

$$
\left(c^{p} \smile c^{q}\right) \smile c^{r}=c^{p} \smile\left(c^{q} \smile c^{r}\right) .
$$

The digital simplicial cochain given by $1_{X}$ is the unit element, that is,

$$
1_{X} \smile c^{p}=c^{p} \smile 1_{X}=c^{p} .
$$

Theorem 45[23] If $\quad c^{p} \in H^{p, \kappa}\left(X, G_{1}\right) \quad$ and $c^{q} \in H^{q, \kappa}\left(X, G_{2}\right)$ are digital cocycles, then

$$
c^{p} \smile c^{q}=(-1)^{p q} c^{q} \smile c^{p} .
$$

Theorem 46[23] Let $\left(X, \kappa_{1}\right) \subset \mathbb{Z}^{n_{1}}$ and $\left(Y, \kappa_{2}\right) \subset \mathbb{Z}^{n_{2}}$ be digital images. If $f:\left(X, \kappa_{1}\right) \rightarrow\left(Y, \kappa_{2}\right)$ is a digitally continuous map and $c^{p} \in H^{p, \kappa}\left(X, G_{1}\right)$ and $c^{q} \in H^{q, \kappa}\left(X, G_{2}\right)$ are digital cocycles, then

$$
f^{*}\left(c^{p} \smile c^{q}\right)=f^{*}\left(c^{p}\right) \smile f^{*}\left(c^{q}\right) .
$$

Definition 47[22] Let $(X, \kappa)$ be a digital simplicial complex. $H^{*, \kappa}(X ; G)=\oplus H^{i, \kappa}(X ; G)$ is the ring with the cup product. This is called the digital simplicial cohomology ring of $X$.

Example 48Consider $M S S_{18}^{\prime}$.

$$
H^{q, 18}\left(M S S_{18}^{\prime}\right)=\left\{\begin{array}{l}
\mathbb{Z}, q=0,2 \\
0, q \neq 0,2
\end{array}\right.
$$

From Example 2.21, 1-cocycles of simplicial complex are

$$
\begin{aligned}
& \omega=e_{0}^{*}+e_{1}^{*}+e_{2}^{*}+e_{3}^{*}, \\
& z=-e_{2}^{*}+e_{4}^{*}-e_{5}^{*}+e_{6}^{*}, \\
& \alpha=-e_{3}^{*}+e_{7}^{*}-e_{8}^{*}+e_{9}^{*}, \\
& \beta=-e_{0}^{*}-e_{4}^{*}-e_{7}^{*}+e_{10}^{*}, \\
& \gamma=-e_{1}^{*}+e_{5}^{*}+e_{8}^{*}+e_{11}^{*}, \\
& \delta=-e_{6}^{*}-e_{9}^{*}-e_{10}^{*}-e_{11}^{*} .
\end{aligned}
$$

Let's compute the cup product of 1-cocycles $\omega, z, \alpha$, $\beta$, $\gamma$ and $\delta$ :

$$
\begin{aligned}
\left\langle\omega \smile z, \sigma_{0}\right\rangle & =\left\langle\omega \smile z,\left[p_{2} p_{4} p_{1}\right]\right\rangle \\
& =\left\langle\omega,\left[p_{2} p_{4}\right]\right\rangle \cdot\left\langle z,\left[p_{4} p_{1}\right]\right\rangle=1.1=1, \\
\left\langle\omega \smile \alpha, \sigma_{4}\right\rangle & =\left\langle\omega \smile \alpha,\left[p_{2} p_{5} p_{1}\right]\right\rangle \\
& =\left\langle\omega,\left[p_{2} p_{5}\right]\right\rangle \cdot\left\langle\alpha,\left[p_{5} p_{1}\right]\right\rangle=1.1=1,
\end{aligned}
$$



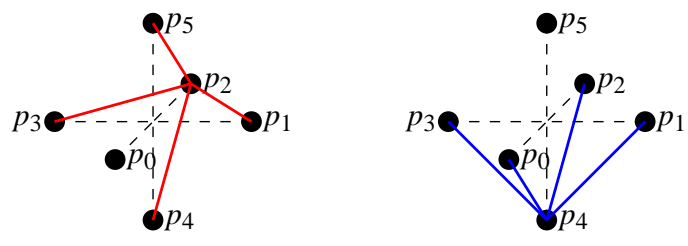

Fig. 8: Cocycle $\omega$ and cocycle $z$
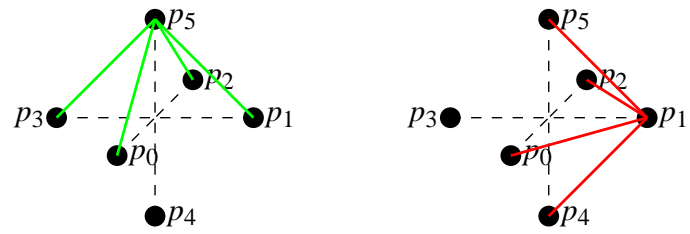

Fig. 9: Cocycle $\alpha$ and cocycle $\beta$
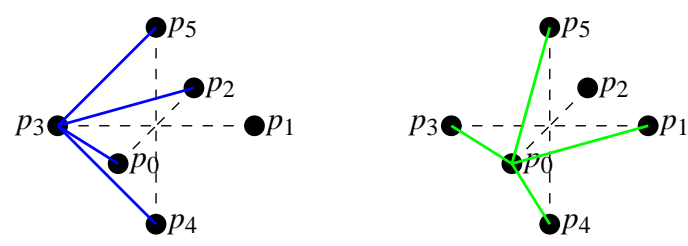

Fig. 10: Cocycle $\gamma$ and cocycle $\delta$

$$
\begin{aligned}
\left\langle\omega \smile \beta, \sigma_{0}\right\rangle & =\left\langle\omega \smile \beta,\left[p_{2} p_{4} p_{1}\right]\right\rangle \\
& =\left\langle\omega,\left[p_{2} p_{5}\right]\right\rangle \cdot\left\langle\beta,\left[p_{5} p_{1}\right]\right\rangle=1 .(-1)=-1, \\
\left\langle\omega \smile \gamma, \sigma_{3}\right\rangle & =\left\langle\omega \smile \gamma,\left[p_{2} p_{3} p_{4}\right]\right\rangle \\
& =\left\langle\omega,\left[p_{2} p_{3}\right]\right\rangle \cdot\left\langle\gamma,\left[p_{3} p_{4}\right]\right\rangle=1.1=1, \\
\left\langle\omega \smile \delta, \sigma_{0}\right\rangle & =\left\langle\omega \smile \delta,\left[p_{2} p_{4} p_{1}\right]\right\rangle \\
& =\left\langle\omega,\left[p_{2} p_{4}\right]\right\rangle \cdot\left\langle\delta,\left[p_{4} p_{1}\right]\right\rangle=1.0=0, \\
\left\langle\omega \smile \omega, \sigma_{0}\right\rangle & =\left\langle\omega \smile \omega,\left[p_{2} p_{4} p_{1}\right]\right\rangle \\
& =\left\langle\omega,\left[p_{2} p_{4}\right]\right\rangle \cdot\left\langle\omega,\left[p_{4} p_{1}\right]\right\rangle=1.0=0 .
\end{aligned}
$$

After calculating cup product of other cocycles, we obtain the follow table.

\begin{tabular}{|c|c|c|c|c|c|c|}
\hline & $\omega$ & $z$ & $\alpha$ & $\beta$ & $\gamma$ & $\delta$ \\
\hline$\omega$ & 0 & 1 & 1 & -1 & 1 & 0 \\
\hline$z$ & 0 & -1 & 0 & 1 & 0 & -1 \\
\hline$\alpha$ & 0 & 0 & -1 & 1 & 0 & 1 \\
\hline$\beta$ & 0 & 0 & 0 & -1 & 0 & 1 \\
\hline$\gamma$ & 0 & 1 & 1 & 0 & -1 & -1 \\
\hline$\delta$ & 0 & 0 & 0 & 0 & 0 & 0 \\
\hline
\end{tabular}

Example 49Consider $\mathrm{MSS}_{18}$.

$$
H^{q, 18}\left(M_{\left.S S_{18}\right)}=\left\{\begin{array}{r}
\mathbb{Z}^{3}, q=0 \\
\mathbb{Z}^{3}, q=1 \\
0, q \geq 2
\end{array}\right.\right.
$$

By example 222, we obtain 1-cocycles of simplicial complex:

$$
\begin{array}{ll}
x=e_{0}^{*}+e_{1}^{*}+e_{7}^{*}+e_{6}^{*}, & a=e_{13}^{*}+e_{15}^{*}+e_{18}^{*}+e_{19}^{*}, \\
y=e_{0}^{*}+e_{5}^{*}+e_{3}^{*}+e_{9}^{*}, & b=e_{16}^{*}+e_{17}^{*}-e_{19}^{*}+e_{12}^{*}, \\
z=e_{2}^{*}-e_{3}^{*}+e_{6}^{*}-e_{7}^{*}, & c=e_{14}^{*}+e_{15}^{*}+e_{16}^{*}+e_{12}^{*}, \\
r=e_{1}^{*}-e_{2}^{*}-e_{5}^{*}+e_{9}^{*}, & d=e_{14}^{*}-e_{17}^{*}+e_{19}^{*}-e_{16}^{*}, \\
w=e_{0}^{*}+e_{1}^{*}+e_{3}^{*}-e_{2}^{*}, & e=e_{14}^{*}+e_{15}^{*}-e_{17}^{*}+e_{19}^{*}, \\
k=e_{1}^{*}-e_{5}^{*}+e_{6}^{*}, & f=e_{14}^{*}-e_{13}^{*}+e_{12}^{*}, \\
l=e_{3}^{*}-e_{7}^{*}+e_{9}^{*}, & g=e_{15}^{*}+e_{13}^{*}+e_{16}^{*}, \\
u=e_{0}^{*}+e_{5}^{*}+e_{7}^{*}, & h=e_{17}^{*}+e_{12}^{*}+e_{18}^{*}, \\
v=e_{2}^{*}+e_{6}^{*}+e_{9}^{*}, & i=e_{19}^{*}-e_{16}^{*}+e_{18}^{*} .
\end{array}
$$



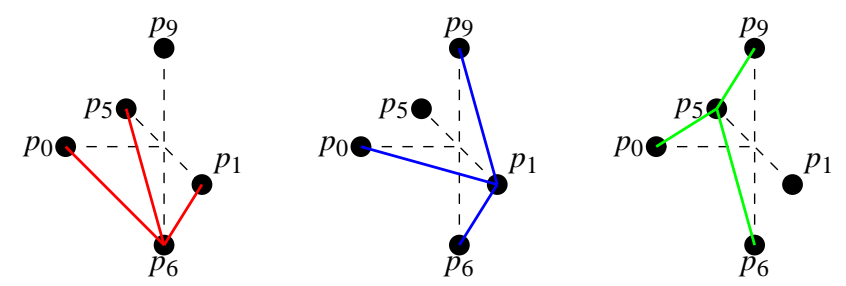

Fig. 13: Cocycle $l$, cocycle $u$ and cocycle $v$

$$
\begin{aligned}
& =\left\langle x,\left[p_{0} p_{9}\right]\right\rangle \cdot\left\langle z,\left[p_{9} p_{1}\right]\right\rangle=1.0=0, \\
\left\langle x \smile r, \sigma_{0}\right\rangle & =\left\langle x \smile r,\left[p_{0} p_{9} p_{1}\right]\right\rangle \\
& =\left\langle x,\left[p_{0} p_{9}\right]\right\rangle \cdot\left\langle r,\left[p_{9} p_{1}\right]\right\rangle=1 .(-1)=-1, \\
\left\langle x \smile w, \sigma_{2}\right\rangle & =\left\langle x \smile w,\left[p_{5} p_{0} p_{6}\right]\right\rangle \\
& =\left\langle x,\left[p_{5} p_{0}\right]\right\rangle \cdot\left\langle w,\left[p_{0} p_{6}\right]\right\rangle=0.1=0, \\
\left\langle x \smile k, \sigma_{0}\right\rangle & =\left\langle x \smile k,\left[p_{0} p_{9} p_{1}\right]\right\rangle \\
& =\left\langle x,\left[p_{0} p_{9}\right]\right\rangle \cdot\left\langle k,\left[p_{9} p_{1}\right]\right\rangle=1 .(-1)=-1, \\
\left\langle x \smile l, \sigma_{2}\right\rangle & =\left\langle x \smile l,\left[p_{5} p_{0} p_{6}\right]\right\rangle \\
& =\left\langle x,\left[p_{5} p_{0}\right]\right\rangle \cdot\left\langle l,\left[p_{0} p_{6}\right]\right\rangle=0.1=0, \\
\left\langle x \smile u, \sigma_{0}\right\rangle & =\left\langle x \smile u,\left[p_{0} p_{9} p_{1}\right]\right\rangle \\
& =\left\langle x,\left[p_{0} p_{9}\right]\right\rangle \cdot\left\langle u,\left[p_{9} p_{1}\right]\right\rangle=1.1=1, \\
\left\langle x \smile v, \sigma_{0}\right\rangle & =\left\langle x \smile v,\left[p_{0} p_{9} p_{1}\right]\right\rangle \\
& =\left\langle x,\left[p_{0} p_{9}\right]\right\rangle \cdot\left\langle v,\left[p_{9} p_{1}\right]\right\rangle=1.0=0 .
\end{aligned}
$$

After calculating cup product of other cocycles, we obtain the following table.

\begin{tabular}{|c|c|c|c|c|c|c|c|c|c|}
\hline & $x$ & $y$ & $z$ & $r$ & $w$ & $k$ & $l$ & $u$ & $v$ \\
\hline$x$ & 0 & 1 & 0 & -1 & 0 & -1 & 0 & 1 & 0 \\
\hline$y$ & 1 & 0 & -1 & 0 & 0 & 0 & -1 & 1 & 0 \\
\hline$z$ & 1 & 1 & 1 & 1 & 1 & 1 & 1 & -1 & 0 \\
\hline$r$ & -1 & 1 & 1 & -1 & -1 & -1 & -1 & 1 & 0 \\
\hline$w$ & 1 & 1 & 1 & -1 & -1 & -1 & -1 & 1 & 0 \\
\hline$k$ & 0 & 1 & 0 & -1 & 0 & -1 & 0 & 1 & 0 \\
\hline$l$ & 1 & 0 & -1 & 0 & 0 & 0 & -1 & 1 & 0 \\
\hline$u$ & 0 & 0 & 0 & 0 & 0 & 0 & 0 & 0 & 0 \\
\hline$v$ & 1 & 1 & -1 & 1 & 1 & 1 & 1 & 0 & 0 \\
\hline
\end{tabular}

\section{Conclusion}

The aim of this paper is to show that some properties from algebraic topology are hold in digital topology. At first relative cohomology groups of digital images are defined and we show that cohomology groups of a digital image are determined the simpler way. Secondly we present that ring structure be existed on the digital simplicial cohomology groups with the cup product. Also we give some examples relevant to computing the cohomology ring of digital images. We expect that these topics will be useful to research digital cohomology operations.

\section{References}

[1] H. Arslan, I. Karaca, and A. Oztel, Homology groups of n-dimensional digital images XXI. Turkish National Mathematics Symposium, B1-13 (2008).

[2] G. Bertrand, Simple points, topological numbers and geodesic neighborhoods in cubic grids, Pattern Recognit. Lett., 15, 1003-1011 (1994).

[3] G. Bertrand, and R. Malgouyres, Some topological properties of discrete surfaces, J. Math. Imaging Vis., 20, 207-221 (1999).

[4] L. Boxer, Digitally continuous functions, Pattern Recognit. Lett., 15, 833-839 (1994).

[5] L. Boxer, A classical construction for the digital fundamental group, J. Math. Imaging Vis., 10, 51-62 (1999).

[6] L. Boxer, Homotopy properties of sphere-like digital images, J. Math. Imaging Vis., 24, 167-175 (2006).

[7] L. Boxer, Digital products, wedges, and covering spaces, J. Math. Imaging Vis., 25, 159-171 (2006).

[8] L. Boxer, Continuous maps on digital simple closed curves, Appl. Math., 1, 377-386 (2010).

[9] L. Boxer, I. Karaca, and A. Oztel, Topological invariants in digital images, J. Math. Sci.:Adv. Appl., 11, 109-140 (2011).

[10] O. Ege and I. Karaca, Cohomology theory for digital images, Romanian Journal of Information Science and Technology, 16, 10-28 (2013).

[11] S.E. Han, Connected sum of digital closed surfaces, Information Sci., 176, 332-348 (2006).

[12] S.E. Han, Minimal simple closed 18-surfaces and a topological preservation of 3D surfaces, Information Sci., 176, 120-134 (2006).

[13] A. Hatcher, Algebraic Topology, Cambridge University Press, (2002).

[14] G.T. Herman, Oriented surfaces in digital spaces, CVGIP: Graph. Models Image Process., 55, 381-396 (1993).

[15] R. Gonzalez-Diaz, and P. Real, On the cohomology of 3D digital images, Discret. Appl. Math., 147, 245-263 (2005).

[16] R. Gonzalez-Diaz, M.J. Jimenez, B. Medrano, Cubical cohomology ring of 3D photographs, Comput. Vis. Pattern Recognit., 21, 76-85 (2011).

[17] R. Gonzalez-Diaz, J. Lamar, and R. Umble, Cup products on polyhedral approximations of 3D digital images, Combinatorial Image Analysis, 6636, 107-119 (2011).

[18] T. Kaczynski, and M. Mrozek, The cubical cohomology ring: an algoritmic approach, Found. Comput. Math. (2012). doi:10.1007/s1020801291384.

[19] T.Y. Kong, A digital fundamental group, Comput. Graph., 13, 159-166 (1989).

[20] R. Kopperman, R. Meyer, R.G. Wilson, A Jordan surface theorem for three-dimensional digital spaces, Discret. Comput. Geo., 6, 155-161 (1991). 
[21] R. Malgouyres and G. Bertrand, A new local property of strong $n$-surfaces, Pattern Recognit. Lett., 20, 417-428 (1999).

[22] J.R. Munkres, Elements of Algebraic Topology, AddisonWesley Publishing Company, (1984).

[23] V.V. Prasolov, Elements of Homology Theory, American Mathematical Society, (2007).

[24] A. Rosenfeld, Continuous functions on digital images, Pattern Recognit. Lett., 4, 177-184 (1986).

[25] J.J. Rotman, An Introduction to Algebraic Topology, Springer-Verlag, New York, (1998).

[26] E. Spanier, Algebraic Topology, McGraw-Hill, New York, (1966).

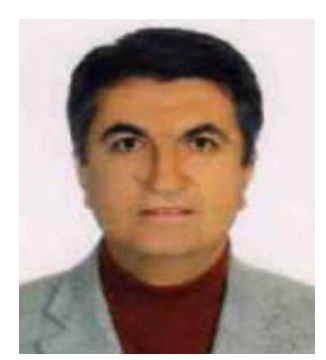

Ismet Karaca was born in Afyon, Turkey on January 5th, 1969. He received a Bachelor's degree in Mathematics from Anadolu University in Turkey, a Master's in Mathematics from the university of Miami, and a $\mathrm{PhD}$ in Mathematics from Lehigh University. $\mathrm{He}$ is a Professor of Mathematics at Ege University in Izmir, TURKEY. Dr. Karaca's research interests include homotopy theory, steenrod algebra, and digital topology. Prof. Karaca is a member of American Mathematical Society.

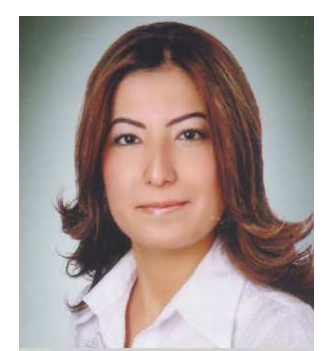

Gulseli Burak was born in Denizli, Turkey on October 26th, 1979. She received a Bachelor's degree in Mathematics from Pamukkale University, a Master's in Mathematics from the same university and she is a integrated $\mathrm{PhD}$ student in the field of digital topology at Adnan Menderes University in Aydin, TURKEY. She is interested in algebraic topology and digital topology. 